\title{
Existence of strong solutions for quasi-static evolution in brittle fracture
}

\author{
JEAN-François BABADJiAn AND ALESSANDRo GiaCOMINi
}

\begin{abstract}
This paper is devoted to prove the existence of strong solutions for a brittle fracture model of quasi-static crack propagation in the two dimensional antiplane setting. As usual, the time continuous evolution is obtained as the limit of a discrete in time evolution by letting the time step tend to zero. The analysis rests on a density lower bound estimate for quasi-minimizers of Mumford-Shah type functionals, under a homogeneous Dirichlet boundary condition on a part of the boundary. In contrast with the previous results, since boundary cracks may be obtained as limits of interior cracks, such a density lower bound has to be established also on balls centered inside the domain but possibly intersecting the Dirichlet boundary. Thanks to a $2 \mathrm{D}$ geometrical argument, the discrete in time crack turns out to satisfy a uniform density lower bound which can pass to the limit, leading to the closedness of the continuous in time crack. We also establish better convergence properties of the discrete in time displacement/crack pair towards its time continuous counterpart.
\end{abstract}

Mathematics Subject Classification (2010): 49Q20 (primary); 35R35, 74R10, 35J70, 49N60 (secondary).

\section{Introduction}

The variational approach to fracture introduced by Francfort and Marigo in [17] (see also [4] and the pioneering paper by Ambrosio and Braides [1]) is now a wellestablished theory for quasi-static crack propagation. It rests on Griffith' original idea that crack propagation is the outcome of the interplay between the elastic energy stored in the material, and the surface energy needed to elongate (or create) a crack.

If $\Omega \subseteq \mathbb{R}^{N}$ is the reference configuration of the body, a configuration of $\Omega$ is given by a pair $(u, \Gamma)$ where $\Gamma \subseteq \Omega$ stands for the crack, and $u: \Omega \backslash \Gamma \rightarrow \mathbb{R}^{N}$ is the associated displacement. Then $(u, \Gamma)$ carries a total energy of the form

$$
\mathcal{E}(u, \Gamma)=\mathcal{E}_{e l}(u, \Gamma)+\mathcal{E}_{s}(\Gamma)
$$

The research of JFB has been supported by the Agence Nationale de la Recherche under Grant No. ANR 10-JCJC 0106.

Received June 8, 2011; accepted in revised form September 4, 2012. 
where

$$
\mathcal{E}_{e l}(u, \Gamma):=\int_{\Omega \backslash \Gamma} W(\nabla u) d x \quad \text { and } \quad \mathcal{E}_{S}(\Gamma)=\kappa \mathcal{H}^{N-1}(\Gamma) .
$$

Here $\mathcal{E}_{e l}(u, \Gamma)$ is the elastic energy associated to the displacement $u$, and $W$ is the elastic energy density of the material: in the case of isotropic linearized elasticity, $W$ is a quadratic function involving the Lamé coefficients of the material. The term $\mathcal{E}_{S}(\Gamma)$ is, according to Griffith' ideas, the energy required to break the material and create the crack $\Gamma$ : within the framework of brittle fracture, $\mathcal{E}_{s}$ is proportional to the surface of $\Gamma$ (here $\mathcal{H}^{N-1}$ stands for the $(N-1)$ dimensional Hausdorff measure, which reduces to the usual notion of area for sufficiently regular sets) by means of a constant $\kappa>0$, usually referred to as the toughness of the material.

The Francfort and Marigo model deals with crack propagation in terms of variational properties of the total energy (1.1). Given a time varying prescribed boundary displacement $[0, T] \ni t \mapsto g(t)$, and in absence of external body and traction forces, a quasi-static crack evolution is - according to [17] - a map $t \mapsto$ $(u(t), \Gamma(t))$ such that $u(t)=g(t)$ on $\partial \Omega$ for every $t \in[0, T]$, and the following properties are satisfied:

(a) Irreversibility: $t \mapsto \Gamma(t)$ is increasing in time, i.e., $\Gamma(s) \subseteq \Gamma(t)$ for every $0 \leq s \leq t \leq T$

(b) Global stability: for every configuration $\left(v, \Gamma^{\prime}\right)$ with $v=g(t)$ on $\partial \Omega$ and $\Gamma(t) \subseteq \Gamma^{\prime}$

$$
\mathcal{E}(u(t), \Gamma(t)) \leq \mathcal{E}\left(v, \Gamma^{\prime}\right)
$$

(c) Energy balance: for every $t \in[0, T]$

$$
\mathcal{E}(u(t), \Gamma(t))=\mathcal{E}(u(0), \Gamma(0))+\int_{0}^{t} \int_{\Omega \backslash \Gamma(s)} D W(\nabla u(s, x)) \cdot \nabla \dot{g}(s, x) d x d s,
$$

where $g(t)$ is extended to $\Omega$ (every extension is admissible, see (1.4) below).

The first condition implies that no healing process can occur during the evolution: the cracking process is irreversible. The second condition states that $(u(t), \Gamma(t))$ is an absolute minimizer of the total energy (1.1) among all admissible configurations for the boundary displacement $g(t)$ and with crack containing $\Gamma(t)$. In particular, by taking $\Gamma^{\prime}=\Gamma(t)$, it turns out that the displacement $u(t)$ minimizes the elastic energy among all admissible displacements at time $t$, i.e. it coincides with the elastic displacement $u_{\Gamma(t)}$ associated to $\Gamma(t)$ and $g(t)$. Under suitable regularity assumptions, $u_{\Gamma(t)}$ satisfies the following elliptic problem

$$
\begin{cases}-\operatorname{div}\left(D W\left(\nabla u_{\Gamma(t)}\right)\right)=0 & \text { in } \Omega \backslash \Gamma(t) \\ u_{\Gamma(t)}=g(t) & \text { on } \partial \Omega \\ D W\left(\nabla u_{\Gamma(t)}\right) v=0 & \text { on } \Gamma(t),\end{cases}
$$


where $v$ denotes the normal to $\Gamma(t)$. Since external body forces are absent, the elastic stress $D W\left(\nabla u_{\Gamma(t)}\right)$ is divergence free, and its normal component on $\Gamma(t)$ vanishes: the crack is thus traction free. Finally the third condition amounts in a sort of energy conservation statement: the variation of the total energy is given by the work done by the external loads, since integrating by parts and taking into account (1.3)

$$
\begin{aligned}
& \int_{\Omega \backslash \Gamma(s)} D W(\nabla u(s, x)) \cdot \nabla \dot{g}(s, x) d x \\
& \quad=\int_{\partial \Omega} D W\left(\nabla u_{\Gamma(s)}(x)\right) v(x) \cdot \dot{g}(s, x) d \mathcal{H}^{N-1}(x),
\end{aligned}
$$

where $\nu(x)$ denotes the outer normal to $\Omega$ at $x \in \partial \Omega$.

Conditions (a)-(c) above retain their meaning even if the admissible configurations enjoy very low regularity properties: namely it is sufficient a notion of differentiability to be defined for the displacements $u$ in order to compute the elastic strain $\nabla u$ and the associated elastic energy. On an elementary level, it suffices that

$$
\Gamma \text { is closed } \quad \text { and } \quad u \in \mathcal{C}^{1}(\Omega \backslash \Gamma) .
$$

As a consequence, the theory can handle geometries for the cracks which are much more general than those covered by the classical Griffith theory, for which, in a two dimensional setting for example, the cracks are curves parametrized by arc length. It has been shown in [17] that if the quasi-static evolutions fit the classical framework, they turn out to satisfy the usual conditions à la Griffith for the propagation involving energy release rates. As a consequence, the Francfort-Marigo model can be considered as a genuine generalization of Griffith' one, in which the crack path is a true unknown of the problem.

The drawback of the theory lies essentially in the issue of global minimality involved in condition (b), i.e., $(u(t), \Gamma(t))$ is an absolute minimizer for the total energy among configurations whose associated crack contains $\Gamma(t)$. Global minimality should rather be replaced by some notion of local minimality which is far from being obvious, since the family of cracks should be endowed with a topology. A suitable notion of local minimality which is useful in this context has been formulated by Larsen in [19].

As suggested in [17], quasi-static evolutions are obtained using variational arguments employing the following scheme.

1. Given a discretization $0=t_{0}^{k}<t_{1}^{k}<\ldots<t_{n(k)}^{k}=T$ of the time interval $[0, T]$, incremental configurations $\left(u_{i}^{k}, \Gamma_{i}^{k}\right)$ at time $t_{i}$ are constructed by minimizing

$$
(u, \Gamma) \mapsto \int_{\Omega \backslash \Gamma_{i-1}^{k}} W(\nabla u) d x+\kappa \mathcal{H}^{N-1}(\Gamma)
$$

among all admissible configurations $(u, \Gamma)$ with $u=g\left(t_{i}^{k}\right)$ on $\partial \Omega$ and $\Gamma \supseteq$ $\Gamma_{i-1}^{k}$. Incremental configurations are readily seen to be globally stable in the sense of (1.2). 
2. A discrete in time evolution $[0, T] \ni t \mapsto\left(u^{k}(t), \Gamma^{k}(t)\right)$ is constructed as the right continuous piecewise constant interpolation in time of $\left\{\left(u_{i}^{k}, \Gamma_{i}^{k}\right): k \in\right.$ $\mathbb{N}, i=0, \ldots, n(k)\}$. It is easily seen that for every $k \in \mathbb{N}$ and $t_{i}^{k} \leq t<t_{i+1}^{k}$, the following energy inequality is satisfied:

$$
\begin{aligned}
\mathcal{E}\left(u^{k}(t), \Gamma^{k}(t)\right) \leq & \mathcal{E}\left(u^{k}(0), \Gamma^{k}(0)\right) \\
& +\int_{0}^{t_{i+1}^{k}} \int_{\Omega} D W\left(\nabla u^{k}(s, x)\right) \cdot \nabla \dot{g}(s, x) d x d s+\varepsilon_{k},
\end{aligned}
$$

where $\varepsilon_{k} \rightarrow 0$.

3. A quasi-static evolution is then obtained formally by letting the time step discretization vanish, and considering the limit, in a suitable sense, of the discrete in time evolutions. Indeed, assuming that global stability and the previous energy inequality are preserved in the limit, the opposite inequality necessary for the energy balance is easily established.

The problem of finding a rigorous mathematical framework for the above mentioned procedure has been addressed in many works. In order to formulate rigorous existence results for incremental configurations, one is led to specify the structure of the cracks and the displacements. Moreover, in order to employ the direct method of the calculus of variations, one needs to conveniently "topologize" the family of admissible configurations. As a consequence, it turns out that the Dirichlet condition has to be reformulated since, within this scheme, cracks can naturally reach the boundary. A convenient way to solve the problem is that of assuming $\Gamma \subseteq \bar{\Omega}$, and prescribing the boundary displacement only on $\partial \Omega \backslash \Gamma$ : no displacement is transmitted on those parts of the boundary which are "broken".

The first mathematical result in this direction is due to Dal Maso and Toader [11]. In their paper, a 2D linearized antiplane setting is considered, for which one can assume $W(\nabla u)=|\nabla u|^{2}$. The cracks are supposed to be closed sets with an a priori fixed number of connected components and with finite $\mathcal{H}^{1}$-measure. The displacements are assumed to be Sobolev regular outside the crack. The minimum problems (1.6) are interpreted as problems involving only $\Gamma$, i.e.,

$$
\Gamma \mapsto\left\|\nabla u_{\Gamma}\right\|_{2}^{2}+\kappa \mathcal{H}^{1}(\Gamma),
$$

where $u_{\Gamma}$ is the elastic displacement associated to $\Gamma$ and $g\left(t_{i}^{k}\right)$. The bound on the number of the connected components makes the functional (1.7) lower semicontinuous with respect to the Hausdorff convergence of compact sets (see Section 2 for a definition). Indeed the surface energy is lower semicontinuous in view of Golab's theorem (see e.g. [14]), and at the same time the elastic energy turns out to be continuous in view of stability results for the solutions of Neumann problems on varying domains (see also [5] for a more general result in this direction). The same method has been employed by Chambolle [7] to treat the case of plane elasticity.

Subsequently, the result has been generalized by Francfort and Larsen [16] to any space dimension. This study imparts from the observation that the model 
of crack propagation presents similarities with that of minimizing the MumfordShah functional arising in image segmentation. They actually proved the existence of weak solutions for this model where the displacement is interpreted as a $S B V$ function. The main tool of that paper is a jump transfer theorem (replacing the stability of the Neumann problems) enabling the authors to pass to the limit in the global stability condition from discrete times to continuous ones. Within this approach the crack $\Gamma(t)$ at time $t$ is actually defined as

$$
\Gamma(t):=\bigcup_{s \in D, s \leq t} S_{u(s)},
$$

where $D \subseteq[0, T]$ is a countable set, and $S_{u(s)}$ is the jump set of $u(s)$. As a consequence, the admissible cracks are countably $\mathcal{H}^{N-1}$-rectifiable sets with finite $\mathcal{H}^{N-1}$-measure.

Then a more general result in the framework of nonlinear elasticity has been proved by Dal Maso, Francfort and Toader [8] for quasi-convex energy densities $W$ with $p$-growth. Here the main difficulty is that the nonconvexity of the problem necessitates one to consider a new notion of weak convergence of cracks (called $\sigma^{p}$-convergence), and the continuous in time cracks are then defined as abstract $\sigma^{p}$-limits of discrete in time ones. However, in [9], it has been proved that, also in that case, the cracks can be described as in (1.8). Finally, Dal Maso and Lazzaroni [10] proved an existence result in the framework of hyper-elasticity for polyconvex energy densities $W$, taking into account the non-interpenetration of matter as well as the fact that the elastic energy should blow up as the determinant of the deformation gradient tends to zero.

The advantage of the weak formulation as in $[8-10,16]$ is that it enables one to prove existence results in very general settings, at the expense of weakening the regularity of the admissible configurations. Indeed the cracks are now rectifiable sets, while the displacements are functions of bounded variation. The object of this paper is to prove that, in the 2-dimensional case, it is possible to prove regularity results for weak solutions. This analysis will be performed thanks to an approach similar to the regularity theory for minimizers of the Mumford-Shah functional (see $[6,12,15])$, leading to the existence of strong solutions.

The idea behind our study is that the discrete in time formulation (1.6) enjoys the same properties than the Mumford-Shah problem. Indeed, starting from a "regular enough" initial datum $\left(u_{0}, \Gamma_{0}\right)$, by iteration and using the direct method in the calculus of variations, it is possible to show the existence of a solution $u_{i}^{k} \in S B V(\Omega)$ to

$$
\begin{aligned}
\min \left\{\int_{\Omega} W(\nabla v) d x+\kappa \mathcal{H}^{N-1}\left(S_{v} \backslash \Gamma_{i-1}^{k}\right):\right. & v \in S B V(\Omega), \\
v & \left.=g\left(t_{i}^{k}\right) \mathcal{H}^{N-1} \text {-a.e. on } \partial \Omega \backslash \Gamma_{i-1}^{k}\right\},
\end{aligned}
$$


where $\Gamma_{i-1}^{k} \subset \Omega$ is the (closed and countably $\mathcal{H}^{N-1}$-rectifiable) crack obtained at the previous time step, and $g\left(t_{i}^{k}\right) \in W^{1, \infty}\left(\mathbb{R}^{N}\right)$ is the updated prescribed boundary displacement at time $t_{i}^{k}$. Then it is possible to apply the regularity results for the Mumford-Shah problem $[6,12,15]$ to ensure that $S_{u_{i}^{k}}$ is (essentially) closed in $\bar{\Omega} \backslash$ $\Gamma_{i-1}^{k}$. The new (closed) crack is then defined by

$$
\Gamma_{i}^{k}:=\Gamma_{i-1}^{k} \cup S_{u_{i}^{k}}
$$

and the associated displacement enjoys a Sobolev regularity outside the crack. Under suitable assumptions on $W$, the equilibrium equation (1.3) entails a $\mathcal{C}^{1}$ regularity for the displacements, yielding thus a discrete in time evolution in a strong sense. Then the main difficulty consists in being able to preserve this property when letting the time step tend to zero.

The regularity results $[6,12,15]$ are based on the fact that the $\mathcal{H}^{N-1}$-density of jump set $S_{u_{i}^{k}}$ at any discontinuity point $x_{0} \in S_{u_{i}^{k}}$ is bounded away from zero. Unfortunately, the density lower bound is not uniform with respect to the time step so that these results are not robust enough to pass directly to the limit. Thus, we need to go deeply into the details of the proofs to find, if possible, uniform estimates. It turns out that this is possible in the $2 \mathrm{D}$ case (see Remark 5.6) for the simple geometrical fact that $\rho \mapsto \mathcal{H}^{N-1}\left(\partial B_{\rho}\right) \sim \rho^{N-1}$ is sub-additive only if $N=2$.

Our approach contains a further difficulty. Indeed in $[12,15]$, the authors proved a density lower bound estimate for balls well contained inside $\Omega$, while in [6] a similar estimate was obtained for balls centered on the (Dirichlet portion of the) boundary of $\Omega$. In our case, we actually need to prove such a density lower bound for balls centered inside $\Omega$ but possibly intersecting the boundary. This is due to the fact that cracks on the boundary can be reached as "limits" of cracks lying inside the body (see Remark 5.8): in this case interior estimates cannot be used to infer estimates for the limit crack.

The paper is organized as follows. In Section 2 we present the main notation and recall the tools employed in the paper.

In Section 3 we collect the regularity properties of the minimizers of the free discontinuity problems involved in the analysis. We show (Proposition 3.11) that minimizers with a non-homogeneous Dirichlet boundary condition $g$ are (modulo a translation by $-g$ ) quasi-minimizers under a homogeneous boundary condition (see Definition 3.3). Then we state (Theorem 3.4) a density lower bound for the jump set of quasi-minimizers in the form we will use to deal with quasi-static evolutions. The proof of this result is postponed to Section 6: it rests on a gradient bound estimate for local minimizers of integral functionals under suitable homogeneous Dirichlet conditions (Theorem 3.8) whose proof is given in Section 7 (the estimate is straightforward in the case $W(\nabla u)=|\nabla u|^{2}$, see Remark 3.9).

In Section 4, we recall the results from [8] concerning existence of quasi-static evolutions. In view of our applications, we restrict to a generalized anti-plane setting, for which the framework of [8] reduces to considering displacements of class 
$S B V$, and countably $\mathcal{H}^{N-1}$-rectifiable cracks. The boundary displacement is imposed only on a portion $\partial_{D} \Omega$ of $\partial \Omega$. Rather than a Griffith' type energy, we consider the more general anisotropic surface energy

$$
\mathcal{E}_{s}(\Gamma):=\int_{\Gamma} \kappa\left(\nu_{\Gamma}\right) d \mathcal{H}^{N-1}
$$

depending on the approximate normal $v_{\Gamma}$ to $\Gamma$. This makes no essential difference (with respect to the scheme illustrated for example in [3, Chapter 7]) concerning the proofs of regularity results for the minimizers of the associated free discontinuity problems.

Section 5 contains the main results of the paper. In the two dimensional case $N=2$, and under suitable assumptions on the reference configuration, the elastic energy density $W$ and the boundary displacement $g$, we prove (Theorem 5.2) the existence of regular evolutions, that is such that $\Gamma(t)$ is closed in the relative topology of $\Omega \cup \partial_{D} \Omega$, and $u(t)$ is Sobolev regular and continuous on $\Omega \backslash \Gamma(t)$. This is a consequence of a uniform $\mathcal{H}^{1}$-density lower bound for the cracks of the incremental configurations (Proposition 5.5), which turns out to be stable as the time step discretization vanishes: we remark that the proof of such a uniformity is the only point in the paper which requires the dimension two, the rest of the analysis being $N$-dimensional. Under further assumptions on $W$, regular evolutions are then shown (Theorem 5.11) to be evolutions in a strong sense, that is satisfying (1.5): we thus obtain the generalization of the result in [11] without requiring a uniform bound on the number of the connected components of the admissible cracks.

The two last sections are devoted to prove Theorems 3.4 and 3.8. The proof of Theorem 3.4 in Section 6 is performed as in [3, Chapter 7], and rests on a decay lemma (Lemma 6.6). Its basic idea is that if the density of jump set of a quasiminimizer $u$ is small enough, then $u$ is closed to being the local minimizer of the bulk energy without jump. As mentioned above, the main point in our analysis is the presence of a homogeneous Dirichlet boundary condition. This reflects on the fact that the local minimality involves a Dirichlet condition on a flat boundary. The precise gradient bound estimate for these local minimizers, Theorem 3.8, is proved by resorting to De Giorgi's approach to the regularity of solutions of nonlinear elliptic partial differential equations.

ACKNOWLEDGEMENTS. The authors acknowledges the University of Brescia for the hospitality and support during the preparation of this paper. Finally the authors wish to thank Massimiliano Morini for interesting discussions on the topic of this paper.

\section{Preliminaries}

In this section we state the main notation and recall the basic definitions employed throughout the paper. In the sequel, $\Omega$ stands for a bounded open subset of $\mathbb{R}^{N}$. 
General notation. We will denote by $B_{\rho}(x)$ the open ball in $\mathbb{R}^{N}$ of center $x \in \mathbb{R}^{N}$ and radius $\rho>0$. If $x=0$, we simply write $B_{\rho}$ instead of $B_{\rho}(0)$.

If $p \geq 1$, we use standard notations for Lebesgue spaces $L^{p}(\Omega)$ and Sobolev spaces $W^{\overline{1}, p}(\Omega)$. We write $|A|$ for the Lebesgue measure of a measurable set $A \subseteq$ $\mathbb{R}^{N}$. Moreover $\omega_{N}:=\left|B_{1}\right|$ will stand for the volume of the unit ball. Finally we will denote by $\mathcal{H}^{N-1}$ the $(N-1)$-dimensional Hausdorff measure.

$S B V$-functions. The space $S B V(\Omega)$ of special functions of bounded variation is given by all functions $u \in L^{1}(\Omega)$ such that the distributional derivative $D u$ of $u$ can be represented as a vector valued bounded Radon measure of the form

$$
D u=\nabla u \mathcal{L}^{N}+\left(u^{+}-u^{-}\right) v_{u} \mathcal{H}^{N-1}\left\llcorner S_{u} .\right.
$$

Here $\mathcal{L}^{N}$ is the Lebesgue measure, $\nabla u \in L^{1}\left(\Omega ; \mathbb{R}^{N}\right)$ is the approximate gradient of $u$, and $S_{u}$ is the jump set of $u$. $S_{u}$ turns out to be countably $\mathcal{H}^{N-1}$-rectifiable, i.e., it is contained up to a set of $\mathcal{H}^{N-1}$-measure zero in the union of $\mathcal{C}^{1}$-submanifolds of $\mathbb{R}^{N}$. It is possible to define $\mathcal{H}^{N-1}$-a.e. on $S_{u}$ an approximate normal denoted by $v_{u}$, as well as traces $u^{ \pm}$. We refer to [3] for a detailed description of that space.

The space $S B V^{p}(\Omega)$, for $p>1$, is a subset of $S B V(\Omega)$ made of all functions $u \in S B V(\Omega)$ such that $\nabla u \in L^{p}\left(\Omega ; \mathbb{R}^{N}\right)$ and $\mathcal{H}^{N-1}\left(S_{u}\right)<+\infty$. One of the main interests of the space $S B V^{p}(\Omega)$ is that Mumford-Shah like functionals are coercive and lower semicontinous in that space according to Ambrosio's compactness Theorem (see [3, Theorems 4.7 and 4.8]).

Theorem 2.1. Let $\left(u_{n}\right) \subset S B V^{p}(\Omega)$ be a sequence such that

$$
\sup _{n \in \mathbb{N}}\left(\left\|u_{n}\right\|_{\infty}+\left\|\nabla u_{n}\right\|_{p}+\mathcal{H}^{N-1}\left(S_{u_{n}}\right)\right)<+\infty .
$$

Then, there exist a subsequence $\left(u_{n_{k}}\right)$ and a function $u \in S B V^{p}(\Omega)$ such that $u_{n_{k}} \rightarrow u$ strongly in $L^{1}(\Omega), \nabla u_{n_{k}} \rightarrow \nabla u$ weakly in $L^{p}\left(\Omega ; \mathbb{R}^{N}\right)$, and

$$
\mathcal{H}^{N-1}\left(S_{u}\right) \leq \liminf _{k \rightarrow+\infty} \mathcal{H}^{N-1}\left(S_{u_{n_{k}}}\right) .
$$

The previous result suggests to define a notion of "weak convergence" in $S B V^{p}(\Omega)$.

Definition 2.2. Let $\left(u_{n}\right) \subset S B V^{p}(\Omega)$ and $u \in S B V^{p}(\Omega)$. We say that $u_{n}$ converges weakly to $u$ in $S B V^{p}(\Omega)$, and we write $u_{n} \rightarrow u$, if $u_{n} \rightarrow u$ strongly in $L^{1}(\Omega), \nabla u_{n} \rightarrow \nabla u$ weakly in $L^{p}\left(\Omega ; \mathbb{R}^{N}\right)$, and $\sup _{n \in \mathbb{N}} \mathcal{H}^{N-1}\left(S_{u_{n}}\right)<+\infty$.

We will use the following semicontinuity property (see [3, Theorem 5.22]): if $u_{n} \rightarrow u$ weakly in $S B V^{p}(\Omega)$, then

$$
\int_{S_{u} \cap A} \kappa\left(v_{u}\right) d \mathcal{H}^{N-1} \leq \liminf _{n \rightarrow+\infty} \int_{S_{u_{n}} \cap A} \kappa\left(v_{u_{n}}\right) d \mathcal{H}^{N-1}
$$

for every norm $\kappa: \mathbb{R}^{N} \rightarrow[0,+\infty)$ and every open set $A \subseteq \Omega$. 
$\sigma^{p}$-convergence of rectifiable sets. We use the notation $\widetilde{\subset}$ (respectively $\cong$ ) for the inclusion (respectively equality) of sets, up to a set of zero $\mathcal{H}^{N-1}$-measure. Let us consider

$\mathcal{R}(\bar{\Omega}):=\left\{\Gamma \widetilde{\subset} \bar{\Omega}: \Gamma\right.$ is countably $\mathcal{H}^{N-1}$-rectifiable with $\left.\mathcal{H}^{N-1}(\Gamma)<+\infty\right\}$.

Note that any $\Gamma \in \mathcal{R}(\bar{\Omega})$ admits at $\mathcal{H}^{N-1}$-a.e. $x \in \Gamma$ an approximate normal denoted by $\nu_{\Gamma}(x)$.

We next recall a notion of convergence for $\mathcal{H}^{N-1}$-rectifiable sets introduced in [8, Definition 4.1] called $\sigma^{p}$-convergence. It is closely related to the jump sets of weakly converging sequences in $S B V^{p}$. Let us fix an open set $\Omega_{0} \subseteq \mathbb{R}^{N}$ such that $\bar{\Omega} \subset \Omega_{0}$.

Definition 2.3. We say that a sequence $\left(\Gamma_{n}\right) \sigma^{p}$-converges in $\bar{\Omega}$ to some $\Gamma \in \mathcal{R}(\bar{\Omega})$ if $\mathcal{H}^{N-1}\left(\Gamma_{n}\right)$ is uniformly bounded, and the following properties are satisfied:

a) if $u_{n_{k}} \rightarrow u$ in $S B V^{p}\left(\Omega_{0}\right)$, and $S_{u_{n_{k}}} \widetilde{\subset} \Gamma_{n_{k}}$, for some sequence $n_{k} \rightarrow+\infty$, then $S_{u} \widetilde{\subset} \Gamma$

b) there exist a function $u \in S B V^{p}\left(\Omega_{0}\right)$ and a sequence $u_{n} \rightarrow u$ in $S B V^{p}\left(\Omega_{0}\right)$, such that $S_{u_{n}} \widetilde{\subset} \Gamma_{n}$ for each $n \in \mathbb{N}$, and $S_{u} \cong \Gamma$.

The $\sigma^{p}$-convergence enjoys good compactness and lower semicontinuity properties as the next result shows (see [8, Theorems 4.3 and 4.7]).

Proposition 2.4. Let $\left(\Gamma_{n}\right)$ be a sequence in $\mathcal{R}(\bar{\Omega})$.

i) If $\mathcal{H}^{N-1}\left(\Gamma_{n}\right)$ is uniformly bounded, then there exist a subsequence $\left(\Gamma_{n_{k}}\right)$ and $\Gamma \in \mathcal{R}(\bar{\Omega})$ such that $\Gamma_{n_{k}} \sigma^{p}$-converges to $\Gamma$ in $\bar{\Omega}$.

ii) If $\Gamma_{n} \sigma^{p}$-converges to some $\Gamma \in \mathcal{R}(\bar{\Omega})$, then

$$
\int_{\Gamma \backslash E} \kappa\left(v_{\Gamma}\right) d \mathcal{H}^{N-1} \leq \liminf _{n \rightarrow+\infty} \int_{\Gamma_{n} \backslash E} \kappa\left(v_{\Gamma_{n}}\right) d \mathcal{H}^{N-1}
$$

for any Borel set $E \subset \bar{\Omega}$ with $\mathcal{H}^{N-1}(E)<+\infty$, and for every norm $\kappa: \mathbb{R}^{N} \rightarrow$ $[0,+\infty)$.

Hausdorff convergence of compact sets. Let $K_{1}$ and $K_{2}$ be compact subsets of $\bar{\Omega}$. The Hausdorff distance between $K_{1}$ and $K_{2}$ is given by

$$
d_{\mathcal{H}}\left(K_{1}, K_{2}\right):=\max \left\{\sup _{x \in K_{1}} \operatorname{dist}\left(x, K_{2}\right), \sup _{y \in K_{2}} \operatorname{dist}\left(y, K_{1}\right)\right\} .
$$

Definition 2.5. We say that a sequence $\left(K_{n}\right)$ of compact subsets of $\bar{\Omega}$ converges in the Hausdorff metric to the compact set $K$ if $d_{\mathcal{H}}\left(K_{n}, K\right) \rightarrow 0$. 
The Hausdorff convergence of compact sets turns out to be equivalent to the convergence in the sense of Kuratowski. Indeed $K_{n} \rightarrow K$ in the Hausdorff metric if and only if both following properties hold:

a) any $x \in K$ is the limit of a sequence $\left(x_{n}\right)$ with $x_{n} \in K_{n}$;

b) if $x_{n} \in K_{n}$, any limit point of $\left(x_{n}\right)$ belongs to $K$.

Finally let us recall the following compactness result (see [3, Theorem 6.1]).

Theorem 2.6 (Blaschke). From any sequence $\left(K_{n}\right)$ of compact subsets of $\bar{\Omega}$, one can extract a subsequence converging in the Hausdorff metric.

\section{Some regularity results for free discontinuity problems}

Let $\Omega \subseteq \mathbb{R}^{N}(N \geq 2), \partial_{D} \Omega \subseteq \partial \Omega, \partial_{N} \Omega \subseteq \partial \Omega$, and $\Omega^{\prime} \subseteq \mathbb{R}^{N}$ be such that

$$
\left\{\begin{array}{l}
\Omega \subseteq \mathbb{R}^{N} \text { is a bounded open set with Lipschitz boundary, } \\
\partial_{D} \Omega \subseteq \partial \Omega \text { is open in the relative topology of } \partial \Omega, \\
\partial_{N} \Omega:=\partial \Omega \backslash \partial_{D} \Omega, \\
\Omega^{\prime} \text { is a bounded open set with } \Omega \subseteq \Omega^{\prime}, \Omega^{\prime} \cap \partial \Omega=\partial_{D} \Omega, \\
\text { and } \operatorname{diam}\left(\Omega^{\prime}\right) \leq 2 \operatorname{diam}(\Omega) .
\end{array}\right.
$$

Let us consider a continuous function $W: \mathbb{R}^{N} \rightarrow \mathbb{R}$ satisfying:

$\left(\mathrm{H}_{1}\right)$ There exist $L>0$ and $p>1$ such that for every $\xi \in \mathbb{R}^{N}$,

$$
L^{-1}|\xi|^{p} \leq W(\xi) \leq L|\xi|^{p}
$$

$\left(\mathrm{H}_{2}\right)$ There exists $\mu>0$ such that for every $\varphi \in \mathcal{C}_{c}^{1}\left(B_{1}\right)$ and $\xi \in \mathbb{R}^{N}$,

$$
\int_{B_{1}}[W(\xi+\nabla \varphi)-W(\xi)] d x \geq \mu \int_{B_{1}}\left(|\xi|^{2}+|\nabla \varphi|^{2}\right)^{\frac{p-2}{2}}|\nabla \varphi|^{2} d x
$$

$\left(\mathrm{H}_{3}\right)$ For every $t>0$ and $\xi \in \mathbb{R}^{N}$,

$$
W(t \xi)=t^{p} W(\xi) .
$$

Remark 3.1. It can been proved that if $W$ is a continuous function satisfying the $p$ growth and $p$-coercivity conditions $\left(\mathrm{H}_{1}\right)$, then the following statements are equivalent:

i) $W$ satisfies $\left(\mathrm{H}_{2}\right)$;

ii) $W$ is uniformly convex, i.e., there exists $\mu_{0}>0$ such that for every $\xi_{1}, \xi_{2} \in$ $\mathbb{R}^{N}$,

$$
W\left(\frac{\xi_{1}+\xi_{2}}{2}\right) \leq \frac{1}{2} W\left(\xi_{1}\right)+\frac{1}{2} W\left(\xi_{2}\right)-\mu_{0}\left(\left|\xi_{1}\right|^{2}+\left|\xi_{2}\right|^{2}\right)^{\frac{p-2}{2}}\left|\xi_{1}-\xi_{2}\right|^{2}
$$


iii) $W(\xi)=c|\xi|^{p}+\psi(\xi)$ for some constant $c>0$, and some convex function $\psi: \mathbb{R}^{N} \rightarrow \mathbb{R}$ satisfying $0 \leq \psi(\xi) \leq L|\xi|^{p}$.

Finally let $\kappa: \mathbb{R}^{N} \rightarrow \mathbb{R}$ be such that

$$
\left\{\begin{array}{l}
\kappa \text { is a norm, } \\
\text { there exists } \beta>0 \text { such that for every } \nu \in \mathbb{R}^{N}, \beta^{-1}|\nu| \leq \kappa(\nu) \leq \beta|\nu|
\end{array}\right.
$$

For every open set $A \subseteq \Omega^{\prime}$ and every $u \in S B V^{p}(A)$, let

$$
F(u, A):= \begin{cases}\int_{A} W(\nabla u) d x+\int_{S_{u} \cap A} \kappa\left(v_{u}\right) d \mathcal{H}^{N-1} & \text { if } u=0 \text { a.e. in } A \cap\left(\Omega^{\prime} \backslash \bar{\Omega}\right), \\ +\infty & \text { otherwise. }\end{cases}
$$

Define also the minimal value

$$
\boldsymbol{m}(u, A):=\inf \left\{F(v, A): v \in S B V^{p}(A),\{v \neq u\} \Subset A\right\},
$$

and the deviation from minimality of $u$ on $A$ by setting, if $\boldsymbol{m}(u, A)<+\infty$,

$$
\operatorname{Dev}(u, A):=F(u, A)-\boldsymbol{m}(u, A) .
$$

We write $F(u)$ for $F\left(u, \Omega^{\prime}\right)$.

Remark 3.2. The role of $\Omega^{\prime}$ in the definition of $F(u, A)$ is that of enforcing in a variational sense the Dirichlet condition on $\partial_{D} \Omega \cap A$ : indeed it is immediately seen that

$$
S_{u} \cap A=\left(S_{u} \cap A \cap \Omega\right) \cup\left\{x \in \partial_{D} \Omega \cap A: u(x) \neq 0\right\},
$$

where the value of $u$ on $\partial_{D} \Omega \cap A$ is intended in the sense of traces. As a consequence, the functional $F(u, A)$ can be described in terms of the behaviour on $A \cap \Omega$ taking into account the value of the traces. We prefer to adopt the setting involving the extended domain $\Omega^{\prime}$ for which points with nonzero traces are treated as ordinary jump points since this is the point of view taken in several papers dealing with quasi-static crack evolutions (see $[8,16]$ ).

In the sequel, we will consider quasi-minimizers of $F$ whose precise definition is given below.

Definition 3.3 (Quasi-minimizers). Let $A \subseteq \Omega^{\prime}$ be an open set. We say that $u \in$ $S B V^{p}(A)$ is a quasi-minimizer of $F(\cdot, A)$ if there exist constants $\omega>0$ and $s \in$ $(0,1)$ such that for every ball $B_{\rho}(x) \subseteq A$

$$
\operatorname{Dev}\left(u, B_{\rho}(x)\right) \leq \omega \rho^{N-1+s} .
$$

Note that the previous definition is not the most general one (see e.g. [2]), but it will be enough for our application. Clearly, if $u$ is a quasi-minimizer of $F(\cdot, A)$, then necessarily $u=0$ a.e. in $\left(\Omega^{\prime} \backslash \bar{\Omega}\right) \cap A$. 
A density lower bound estimate for the jump set of quasi-minimizers of the Mumford-Shah functional, i.e., for $W(\xi)=|\xi|^{2}$ and $\kappa(\nu)=|\nu|$, was shown to hold on balls $B_{\rho}(x) \subseteq \Omega$ by De Giorgi, Carriero and Leaci in [12], and subsequently extended to the nonlinear case (see $[3,15])$. The case of the Mumford-Shah functional with Dirichlet boundary conditions has been treated by Carriero and Leaci in [6], establishing the density lower bound also for balls $B_{\rho}(x)$ with center $x \in \partial_{D} \Omega$. In view of our application to quasi-static crack growth, we need to extend the result to balls possibly intersecting $\partial_{D} \Omega$ but with center inside $\Omega$. This extension is contained in the following theorem, whose proof is postponed to Section 6 .

Theorem 3.4 (Density lower bound for the jump set of quasi-minimizers). Assume that $\Omega$ satisfies (3.1), and that

$$
\partial_{D} \Omega \text { is of class } \mathcal{C}^{1} \text {. }
$$

Let $W: \mathbb{R}^{N} \rightarrow \mathbb{R}$ be a continuous function satisfying $\left(\mathrm{H}_{1}\right)-\left(\mathrm{H}_{3}\right)$, let $A \subseteq \Omega^{\prime}$ be an open set, and let $u \in S B V^{p}(A)$ be a quasi-minimizer of $F(\cdot, A)$ with constants $\omega>0$ and $s \in(0,1)$.

Then for every $\eta>0$, there exist $\vartheta_{0}>0$ and $\rho_{0}>0$ (depending only on $N, p$, $L, \mu, \beta, s, \omega$, and $\eta)$ such that

$$
\mathcal{H}^{N-1}\left(S_{u} \cap B_{\rho}(x)\right) \geq \vartheta_{0} \rho^{N-1}
$$

for all balls $B_{\rho}(x) \subseteq A$ with center $x \in \overline{S_{u}}$, radius $\rho \leq \rho_{0}$ and such that $\operatorname{dist}\left(B_{\rho}(x), \partial_{N} \Omega\right)>\eta$.

The safety parameter $\eta$ is introduced for technical reasons connected to the fact that only the portion $\partial_{D} \Omega$ of $\partial \Omega$ is assumed to be of class $\mathcal{C}^{1}$. This parameter enforces the balls involved in the density lower bound to be well distant from $\partial_{N} \Omega$ on which only Lipschitz regularity is available. Note that if the entire boundary $\partial \Omega$ was of class $\mathcal{C}^{1}$, then it would be useless to introduce $\eta$.

It is essential for our application to crack evolution that the constants $\vartheta_{0}$ and $\rho_{0}$ are independent of the subset $A$ of $\Omega^{\prime}$. Indeed we will need to consider a sequence of minimization problems stated on a decreasing family of open subsets of $\Omega^{\prime}$, and we will need to ensure that the constants obtained in the density lower bound estimates are indeed uniform.

Remark 3.5. Let $u \in S B V^{p}(A)$ be a quasi-minimizer of $F(\cdot, A)$ for $A \subseteq \Omega^{\prime}$. Clearly $S_{u} \subseteq\left(\Omega \cup \partial_{D} \Omega\right) \cap A$. From standard properties of densities (see $e . g$. [3, Section 2.9]), we know that for $\mathcal{H}^{N-1}$-a.e. $x \in\left[\left(\Omega \cup \partial_{D} \Omega\right) \cap A\right] \backslash S_{u}$ one has

$$
\lim _{\rho \rightarrow 0} \frac{\mathcal{H}^{N-1}\left(S_{u} \cap B_{\rho}(x)\right)}{\rho^{N-1}}=0 .
$$

As a consequence of Theorem 3.4 we deduce that

$$
\mathcal{H}^{N-1}\left(\left[\left(\Omega \cup \partial_{D} \Omega\right) \cap A\right] \cap\left(\overline{S_{u}} \backslash S_{u}\right)\right)=0,
$$

so that $S_{u}$ is essentially closed in $\left[\Omega \cup \partial_{D} \Omega\right] \cap A$. 
The proof of Theorem 3.4 relies on a gradient bound estimate for local minimizers of the bulk energy under a homogeneous Dirichlet boundary condition, where the Dirichlet boundary $\partial_{D} \Omega$ has been flattened into the boundary of a half plane. Let us introduce, for $\delta \geq 0$,

$$
H_{\delta}:=\left\{x=\left(x^{\prime}, x_{N}\right) \in \mathbb{R}^{N}: x_{N}>-\delta\right\},
$$

and for every $r \leq 1$ and $u \in W^{1, p}\left(B_{r}\right)$, let

$$
F_{0, \delta}\left(u, B_{r}\right):= \begin{cases}\int_{B_{r}} W(\nabla u) d x & \text { if } u=0 \text { a.e. in } B_{r} \backslash H_{\delta}, \\ +\infty & \text { otherwise. }\end{cases}
$$

Note that if $r<\delta$, then the functional $F_{0, \delta}\left(\cdot, B_{r}\right)$ does not see the Dirichlet condition which therefore becomes irrelevant.

Definition 3.6 (Local minimizers). Let $r>0$. We say that $u \in W^{1, p}\left(B_{r}\right)$ is a local minimizer of $F_{0, \delta}\left(\cdot, B_{r}\right)$ if

$$
F_{0, \delta}\left(u, B_{r}\right) \leq F_{0, \delta}\left(v, B_{r}\right)
$$

for every $v \in W^{1, p}\left(B_{r}\right)$ with $\{u \neq v\} \Subset B_{r}$.

In order to estimate the gradient of such local minimizers we will need to distinguish the cases $\delta>1 / 2$ and $\delta \in[0,1 / 2]$. In the former case, the problem will essentially be reduced to an interior estimate as in [15, Theorem 2.2], while in the latter case, the presence of the flat boundary will play a crucial role in the estimate.

Theorem 3.7 (Interior gradient bound). Assume that $\delta>1 / 2$ and that $W: \mathbb{R}^{N} \rightarrow$ $\mathbb{R}$ is a continuous function satisfying $\left(\mathrm{H}_{1}\right)$ and $\left(\mathrm{H}_{2}\right)$. Let $u \in W^{1, p}\left(B_{1 / 2}\right)$ be a local minimizer of $F_{0, \delta}\left(\cdot, B_{1 / 2}\right)$. Then, there exists a constant $C_{0}=C_{0}(N, p, L, \mu)>0$ such that

$$
\underset{B_{\rho / 2}}{\operatorname{essup}}|\nabla u|^{p} \leq \frac{C_{0}}{\rho^{N}} \int_{B_{\rho}}|\nabla u|^{p} d x \quad \text { for every } \rho \leq 1 / 2 .
$$

Theorem 3.7 is a direct consequence of [15, Theorem 2.2] since $B_{1 / 2} \Subset H_{\delta}$, so that the flat boundary $\partial H_{\delta}$ is not seen by the functional $F_{0, \delta}\left(\cdot, B_{1 / 2}\right)$.

In Section 7, we will prove the following result which extends Theorem 3.7 up to the flat boundary.

Theorem 3.8 (Boundary gradient bound). Assume that $\delta \in[0,1 / 2]$ and that $W$ : $\mathbb{R}^{N} \rightarrow \mathbb{R}$ is a continuous function satisfying $\left(\mathrm{H}_{1}\right)$ and $\left(\mathrm{H}_{2}\right)$. Let $u \in W^{1, p}\left(B_{1}\right)$ be a local minimizer of $F_{0, \delta}\left(\cdot, B_{1}\right)$. Then, for each $R_{0}<1$, there exists a constant $C_{0}^{\prime}=C_{0}^{\prime}\left(N, p, L, \mu, R_{0},\|\nabla u\|_{p}\right)>0$ (independent of $\delta$ ) such that

$$
\underset{B_{\rho / 2}}{\operatorname{esssup}}|\nabla u|^{p} \leq C_{0}^{\prime}\left(\frac{1}{\rho^{N}} \int_{B_{\rho}}|\nabla u|^{p} d x+1\right) \quad \text { for every } \rho \leq R_{0} .
$$


Without loss of generality, one can assume that the constants $C_{0}$ and $C_{0}^{\prime}$ involved in Theorems 3.7 and 3.8 satisfy

$$
C_{0} \leq C_{0}^{\prime}
$$

Note finally that $C_{0}^{\prime}$ is independent of $\delta$ : this point is essential to establish Theorem 3.4 (see Lemma 6.6).

Remark 3.9 (The case $W(\xi)=|\xi|^{2}$ ). Note that the conclusion of Theorem 3.8 is easily established in the case $W(\xi)=|\xi|^{2}$. Indeed in that case the local minimizer $u \in W^{1,2}\left(B_{1}\right)$ is actually a harmonic function over $B_{1} \cap H_{\delta}$, so that harmonic reflexion across $\partial H_{\delta}$ can be used in order to get the gradient bound as a consequence of the mean value property for harmonic functions.

In the study of the quasi-static crack evolution model, we will consider a sequence of minimization problems for free discontinuity functionals with a non homogeneous Dirichlet boundary condition. In order to infer a density lower bound for the jump set of their minimizers, we associate to them suitable quasi-minimizers of (3.3) and apply Theorem 3.4.

Let us consider an open set $A \subseteq \Omega^{\prime}$, and let $u \in S B V^{p}(A)$ be a solution of

$$
\begin{aligned}
\min \left\{\int_{A} W(\nabla v) d x+\int_{S_{v}} \kappa\left(v_{v}\right) d \mathcal{H}^{N-1}:\right. & v \in S B V^{p}(A), \\
& \left.v=g \text { a.e. in }\left(\Omega^{\prime} \backslash \bar{\Omega}\right) \cap A\right\},
\end{aligned}
$$

where $g \in W^{1, \infty}\left(\Omega^{\prime}\right)$. The following energy upper bound estimate holds:

Lemma 3.10. Assume that (3.1) and (3.2) hold, and that $W: \mathbb{R}^{N} \rightarrow \mathbb{R}$ is a continuous function satisfying $\left(\mathrm{H}_{1}\right)$ and $\left(\mathrm{H}_{2}\right)$. Let $A \subseteq \Omega^{\prime}, g \in W^{1, \infty}\left(\Omega^{\prime}\right)$, and let $u \in S B V^{p}(A)$ be a solution of (3.6). There exists a constant $c_{0}>0$ (depending only on $N, p, L, \beta,\|\nabla g\|_{\infty}, \operatorname{diam}(\Omega)$, and the Lipschitz constant of $\partial \Omega$ ) such that for any ball $B_{\rho}(x) \subseteq A$

$$
\|\nabla u\|_{L^{p}\left(B_{\rho}(x) ; \mathbb{R}^{N}\right)}^{p}+\mathcal{H}^{N-1}\left(S_{u} \cap B_{\rho}(x)\right) \leq c_{0} \rho^{N-1} .
$$

Proof. Let us compare $u$ with $v:=u \chi_{A \backslash\left[\Omega \cap B_{\rho}(x)\right]}$. Observe that $v \in S B V^{p}(A)$ with $v=g$ on $\left(\Omega^{\prime} \backslash \bar{\Omega}\right) \cap A$. Moreover

$$
S_{v} \cap \overline{B_{\rho}(x)} \subset\left[\partial B_{\rho}(x) \cap \Omega\right] \cup\left[\partial_{D} \Omega \cap B_{\rho}(x)\right] .
$$

By the minimality of $u$, since $W$ satisfies $\left(\mathrm{H}_{1}\right)$ and $\kappa$ satisfies (3.2), we infer $(v=$ $u=g$ a.e. in $\left.B_{\rho}(x) \cap\left[\Omega^{\prime} \backslash \bar{\Omega}\right]\right)$

$$
\begin{aligned}
& L^{-1} \int_{B_{\rho}(x)}|\nabla u|^{p} d y+\beta^{-1} \mathcal{H}^{N-1}\left(S_{u} \cap B_{\rho}(x)\right) \\
\leq & L \int_{B_{\rho}(x)}|\nabla v|^{p} d y+\beta \mathcal{H}^{N-1}\left(S_{v} \cap \overline{B_{\rho}(x)}\right) \\
\leq & L\|\nabla g\|_{\infty}^{p} \omega_{N} \rho^{N}+\beta N \omega_{N} \rho^{N-1}+\beta \mathcal{H}^{N-1}\left(\partial_{D} \Omega \cap B_{\rho}(x)\right),
\end{aligned}
$$


so that the result follows by the Lipschitz regularity of $\partial \Omega$, and the fact that $\rho \leq$ $\operatorname{diam}\left(\Omega^{\prime}\right) \leq 2 \operatorname{diam}(\Omega)$.

The following result holds:

Proposition 3.11. Assume that (3.1) and (3.2) hold, and that $W: \mathbb{R}^{N} \rightarrow \mathbb{R}$ is a function of class $\mathcal{C}^{1}$ satisfying $\left(\mathrm{H}_{1}\right)$ and $\left(H_{2}\right)$. Let $A \subseteq \Omega^{\prime}, g \in W^{1, \infty}\left(\Omega^{\prime}\right)$, and let $u \in S B V^{p}(A)$ be a solution of (3.6). Then the function

$$
\hat{u}:=u-g \in S B V^{p}(A)
$$

is a quasi-minimizer of $F(\cdot, A)$ for $s=1 / p$ and $\omega>0$ which only depends on $N$, $p, L, \beta,\|\nabla g\|_{\infty}, \operatorname{diam}(\Omega)$, and the Lipschitz constant of $\partial \Omega$.

Proof. Since $S_{\hat{u}}=S_{u}$, then $\hat{u}$ minimizes

$$
v \mapsto \int_{A} W(\nabla v) d y+\int_{0}^{1} \int_{A} D W(\nabla v+s \nabla g) \cdot \nabla g d y d s+\int_{S_{v} \cap A} \kappa\left(v_{v}\right) d \mathcal{H}^{N-1}
$$

among all $v \in S B V^{p}(A)$ with $v=0$ a.e. in $\left(\Omega^{\prime} \backslash \bar{\Omega}\right) \cap A$. Let $B_{\rho}(x) \subseteq A$ and $v \in S B V^{p}(A)$ with $\{v \neq \hat{u}\} \Subset B_{\rho}(x)$. From the previous minimality property we deduce that

$$
\begin{aligned}
F\left(\hat{u}, B_{\rho}(x)\right) \leq & F\left(v, B_{\rho}(x)\right) \\
& +\int_{0}^{1} \int_{B_{\rho}(x)}(D W(\nabla v+s \nabla g)-D W(\nabla \hat{u}+s \nabla g)) \cdot \nabla g d y d s .
\end{aligned}
$$

By the $p$-growth property $\left(\mathrm{H}_{1}\right)$ and the convexity of $W$ (see Remark 3.1), we deduce that its differential $D W$ satisfies the following $(p-1)$-growth condition: for all $\xi \in \mathbb{R}^{N}$,

$$
|D W(\xi)| \leq c|\xi|^{p-1},
$$

for some constant $c>0$ depending only on $p$ and $L$ (see e.g. [18, Lemma 5.2]). Hence, by Hölder's inequality, we infer that

$$
\begin{aligned}
& \left|\int_{0}^{1} \int_{B_{\rho}(x)} D W(\nabla \hat{u}+s \nabla g) \cdot \nabla g d y d s\right| \\
\leq & c\left(\|\nabla \hat{u}\|_{L^{p}\left(B_{\rho}(x) ; \mathbb{R}^{N}\right)}^{p-1}\|\nabla g\|_{L^{p}\left(B_{\rho}(x) ; \mathbb{R}^{N}\right)}+\|\nabla g\|_{L^{p}\left(B_{\rho}(x) ; \mathbb{R}^{N}\right)}^{p}\right),
\end{aligned}
$$


for some constant $c=c(p, L)>0$. Using Lemma 3.10 and the coercivity property $\left(\mathrm{H}_{1}\right)$, we get that

$$
\begin{aligned}
& \left|\int_{0}^{1} \int_{B_{\rho}(x)} D W(\nabla \hat{u}+s \nabla g) \cdot \nabla g d y d s\right| \\
\leq & c\left(\left(\left(c_{0} L \rho^{N-1}\right)^{(p-1) / p}+\left(\|\nabla g\|_{\infty}^{p} \omega_{N} \rho^{N}\right)^{(p-1) / p}\right)\right. \\
& \left.\left(\|\nabla g\|_{\infty}^{p} \omega_{N} \rho^{N}\right)^{1 / p}+\|\nabla g\|_{\infty}^{p} \omega_{N} \rho^{N}\right) \\
\leq & c_{1} \rho^{N-1+1 / p},
\end{aligned}
$$

for some constant $c_{1}>0$ depending only on $N, p, L, \beta,\|\nabla g\|_{\infty}$, $\operatorname{diam}(\Omega)$, and the Lipschitz constant of $\partial \Omega$.

In order to estimate the other term, we use Young's formula to get that

$$
\begin{aligned}
& \left|\int_{0}^{1} \int_{B_{\rho}(x)} D W(\nabla v+s \nabla g) \cdot \nabla g d y d s\right| \\
\leq & c \int_{B_{\rho}(x)}\left(\varepsilon^{p /(p-1)}|\nabla v|^{p}+\left(\varepsilon^{-p}+1\right)|\nabla g|^{p}\right) d y \\
\leq & c_{2}\left(\varepsilon^{p /(p-1)} F\left(v, B_{\rho}(x)\right)+\left(\varepsilon^{-p}+1\right) \rho^{N}\right),
\end{aligned}
$$

for some constant $c_{2}=c_{2}\left(p, L,\|\nabla g\|_{\infty}\right)>0$ and for $\varepsilon>0$ to be fixed later. Hence gathering (3.7), (3.8) and (3.9), we infer that

$$
F\left(\hat{u}, B_{\rho}(x)\right) \leq\left(1+c_{2} \varepsilon^{p /(p-1)}\right) F\left(v, B_{\rho}(x)\right)+c_{1} \rho^{N-1+1 / p}+c_{2}\left(\varepsilon^{-p}+1\right) \rho^{N} .
$$

Taking the infimum with respect to such $v$ 's leads to

$$
\operatorname{Dev}\left(\hat{u}, B_{\rho}(x)\right) \leq c_{2} \varepsilon^{p /(p-1)} F\left(\hat{u}, B_{\rho}(x)\right)+c_{1} \rho^{N-1+1 / p}+c_{2}\left(\varepsilon^{-p}+1\right) \rho^{N} .
$$

As a consequence, using Lemma 3.10 we obtain that

$$
\operatorname{Dev}\left(\hat{u}, B_{\rho}(x)\right) \leq c_{3}\left(\varepsilon^{p /(p-1)} \rho^{N-1}+\rho^{N-1+1 / p}+\left(\varepsilon^{-p}+1\right) \rho^{N}\right)
$$

where $c_{3}>0$ depends only on $N, p, L, \beta,\|\nabla g\|_{\infty}$, $\operatorname{diam}(\Omega)$, and the Lipschitz constant of $\partial \Omega$. If we choose $\varepsilon=\rho^{1 /\left(p p^{\prime}\right)}$, we finally get that

$$
\operatorname{Dev}\left(\hat{u}, B_{\rho}(x)\right) \leq \omega \rho^{N-1+1 / p},
$$

for some constant $\omega>0$ so that $\hat{u}$ is a quasi-minimizer for $F(\cdot, A)$ with the choice $s=1 / p$. 


\section{Quasi-static crack evolutions}

As mentioned in the Introduction, the Francfort-Marigo model for quasi-static crack propagation introduced in [17] has been addressed from a mathematical point of view in several papers, in order to find suitable frameworks in which rigorous existence results could be proved (see $[8,11,16]$ ).

In the sequel, we present a mathematical formulation of the Francfort-Marigo model which is a sort of compromise between [16] and [8]. Specifically we consider an anti-plane generalized geometry with elastic energy density $W$ of convex type, and an anisotropic Griffith energy for the cracks. Within this framework, we have at our disposal the tools for establishing existence of quasi-static crack evolutions, and those for proving regularity results for the jump set of the minimizers of the associated free discontinuity problems.

The reference configuration. Let $\Omega$ be a bounded open subset of $\mathbb{R}^{N}(N \geq 2)$ with Lipschitz boundary which stands for the reference configuration of an elastic material that can experience cracks. We write $\partial \Omega=\partial_{N} \Omega \cup \partial_{D} \Omega$, where $\partial_{D} \Omega$ denotes the Dirichlet part of the boundary on which the displacement is prescribed, and $\partial_{N} \Omega$ denotes the Neumann part of the boundary which is traction free. We assume that $\partial_{D} \Omega$ is open in the relative topology of $\partial \Omega$.

Admissible configurations. Consider a boundary displacement $g$ given by the trace on $\partial_{D} \Omega$ of a function in $W^{1, p}(\Omega)$. We say that a pair $(u, \Gamma)$ is an admissible configuration for $g$, and we write $(u, \Gamma) \in \mathcal{A}(g)$, if $u \in S B V^{p}(\Omega), \Gamma \in \mathcal{R}(\bar{\Omega})$ (see (2.2)) with $S_{u} \widetilde{\subset} \Gamma$, and $u=g \mathcal{H}^{N-1}$-a.e. on $\partial_{D} \Omega \backslash \Gamma$. The set $\Gamma$ stands for the crack of the body, while the function $u$ is the associated displacement.

Bulk and surface energies. Let $W: \mathbb{R}^{N} \rightarrow \mathbb{R}$ be a convex function of class $\mathcal{C}^{1}$ satisfying $\left(\mathrm{H}_{1}\right)$ of Section 3 , and let $\kappa: \mathbb{R}^{N} \rightarrow \mathbb{R}$ be such that (3.2) holds. Given an admissible configuration $(u, \Gamma)$ for the boundary displacement $g$, we set

$$
\mathcal{W}(\nabla u):=\int_{\Omega} W(\nabla u) d x \quad \text { and } \quad \mathcal{K}(\Gamma):=\int_{\Gamma \backslash \partial_{N} \Omega} \kappa\left(\nu_{\Gamma}\right) d \mathcal{H}^{N-1} .
$$

The quantity $\mathcal{W}(\nabla u)$ stands for the elastic energy associated to the displacement $u$, while $\mathcal{K}(\Gamma)$ is the energy associated to the crack $\Gamma$. Following Griffith' ideas, $\mathcal{K}(\Gamma)$ is proportional to the surface of $\Gamma$, and here we consider a possibly anisotropic surface energy depending on the approximate normal $v_{\Gamma}$ to $\Gamma$. Note that $\Gamma \cap \partial_{N} \Omega$ does not contribute to the energy associated to $\Gamma$.

Finally, we define the total energy of the configuration $(u, \Gamma)$ as

$$
\mathcal{E}(u, \Gamma):=\mathcal{W}(\nabla u)+\mathcal{K}(\Gamma) .
$$

Quasi-static evolutions. Let us fix a time interval $[0, T]$, where $T>0$, and consider a time-dependent boundary displacement

$$
g \in A C\left(0, T ; W^{1, p}(\Omega) \cap L^{\infty}(\Omega)\right) .
$$


Definition 4.1 (Quasi-static crack evolution). The map $[0, T] \ni t \mapsto(u(t), \Gamma(t))$ is a quasi-static evolution relative to the boundary displacement $g$ if $(u(t), \Gamma(t)) \in$ $\mathcal{A}(g(t))$ for all $t \in[0, T]$, and if the following properties are satisfied:

(a) Irreversibility: for every $0 \leq s \leq t \leq T$, then $\Gamma(s) \widetilde{\subset} \Gamma(t)$.

(b) Global stability: for every $\left(v, \Gamma^{\prime}\right) \in \mathcal{A}(g(t))$ such that $\Gamma(t) \widetilde{\subset} \Gamma^{\prime}$

$$
\mathcal{E}(u(t), \Gamma(t)) \leq \mathcal{E}\left(v, \Gamma^{\prime}\right) .
$$

(c) Energy balance: for every $t \in[0, T]$, the total energy $\mathcal{E}(t):=\mathcal{E}(u(t), \Gamma(t))$ is absolutely continuous and

$$
\mathcal{E}(t)=\mathcal{E}(0)+\int_{0}^{t} \int_{\Omega} D W(\nabla u(s, x)) \cdot \nabla \dot{g}(s, x) d x d s .
$$

The following result has been proved in [8].

Theorem 4.2. Let $g$ be an admissible boundary displacement satisfying (4.2), and let $\left(u_{0}, \Gamma_{0}\right) \in \mathcal{A}(g(0))$ be globally stable in the sense of (4.3). Then there exists a quasi-static evolution $[0, T] \ni t \mapsto(u(t), \Gamma(t))$ relative to $g$ and such that $(u(0), \Gamma(0))=\left(u_{0}, \Gamma_{0}\right)$.

The previous existence result is obtained following the main ideas outlined in [17]. Namely, a discretized in time evolution is constructed by means of minimum problems taking into account the irreversibility condition which forces the crack to increase in time. Then the time step discretization is sent to zero, and the quasistatic evolution arises as the limit of a suitable piecewise constant interpolation in time of the discretized evolution.

More precisely let

$$
0=t_{0}^{k}<t_{1}^{k}<\cdots<t_{n(k)}^{k}=T
$$

be a discretization of the time interval $[0, T]$ such that the time step

$$
\delta_{k}:=\sup _{1 \leq i \leq n(k)}\left(t_{i}^{k}-t_{i-1}^{k}\right) \rightarrow 0,
$$

and set $g_{i}^{k}:=g\left(t_{i}^{k}\right)$. Setting $\left(u_{0}^{k}, \Gamma_{0}^{k}\right)=\left(u_{0}, \Gamma_{0}\right)$, and assuming to have constructed $\left(u_{i-1}^{k}, \Gamma_{i-1}^{k}\right) \in \mathcal{A}\left(g_{i-1}^{k}\right)$, let $\left(u_{i}^{k}, \Gamma_{i}^{k}\right) \in \mathcal{A}\left(g_{i}^{k}\right)$ be a solution of

$$
\min \left\{\mathcal{W}(\nabla u)+\mathcal{K}(\Gamma):(u, \Gamma) \in \mathcal{A}\left(g_{i}^{k}\right), \Gamma_{i-1}^{k} \widetilde{\subset} \Gamma\right\} .
$$

The existence of solutions to problem (4.6) can be established using the direct method in the calculus of variations (see [8, Theorem 3.10]) thanks to Ambrosio's theorem (Theorem 2.1) and the compactness of $\mathcal{R}(\bar{\Omega})$ with respect to the $\sigma^{p}$ convergence (Proposition 2.4). Note that by a truncation argument we can always assume that for every $i=0, \ldots, n(k)$

$$
\left\|u_{i}^{k}\right\|_{\infty} \leq\left\|g_{i}^{k}\right\|_{\infty} .
$$

For future reference, we need the following definition. 
Definition 4.3 (Incremental configurations and discrete in time evolutions). Consider the discretization (4.4) of the time interval $[0, T]$.

We say that $\left\{\left(u_{i}^{k}, \Gamma_{i}^{k}\right): i=0, \ldots, n(k)\right\}$ is a family of incremental configurations if $\left(u_{i}^{k}, \Gamma_{i}^{k}\right)$ is a solution of (4.6) and (4.7) is satisfied.

The associated discrete in time evolution $[0, T] \ni t \mapsto\left(u^{k}(t), \Gamma^{k}(t)\right)$ is defined as the right continuous piecewise constant interpolations

$$
u^{k}(t):=u_{i}^{k}, \quad g^{k}(t):=g_{i}^{k}, \quad \Gamma^{k}(t):=\Gamma_{i}^{k}, \quad t \in\left[t_{i}^{k}, t_{i+1}^{k}\right),
$$

with $u^{k}(T)=u_{n(k)}^{k}, \Gamma^{k}(T)=\Gamma_{n(k)}^{k}$ and $g^{k}(T)=g_{n(k)}^{k}$.

A quasi-static evolution is obtained from (4.8) by letting $k \rightarrow+\infty$, i.e., by letting the time step discretization tend to zero. More precisely the following result holds (see $[8,16]$ ).

Theorem 4.4. Let $[0, T] \ni t \mapsto\left(u^{k}(t), \Gamma^{k}(t)\right)$ be a discrete in time evolution relative to the discretization $\left\{t_{i}^{k}\right\}_{0 \leq i \leq n(k)}$ of the time interval $[0, T]$ satisfying (4.4) and (4.5). The following properties hold.

(a) There exists a constant $C>0$ such that for every $k \in \mathbb{N}$ and $t \in[0, T]$

$$
\left\|u^{k}(t)\right\|_{\infty}+\left\|\nabla u^{k}(t)\right\|_{p}+\mathcal{H}^{N-1}\left(\Gamma^{k}(t)\right) \leq C .
$$

(b) There exist a quasi-static evolution $[0, T] \ni t \mapsto(u(t), \Gamma(t))$ relative to the boundary displacement $g$ and with initial configuration $\left(u_{0}, \Gamma_{0}\right)$, and a subsequence of $\left(u^{k}(t), \Gamma^{k}(t)\right)$ (not relabelled) such that for every $t \in[0, T]$, $\Gamma(t) \widetilde{\subset} \Omega \cup \partial_{D} \Omega$,

$$
\mathcal{W}\left(\nabla u^{k}(t)\right) \rightarrow \mathcal{W}(\nabla u(t))
$$

and

$$
\mathcal{K}\left(\Gamma^{k}(t)\right) \rightarrow \mathcal{K}(\Gamma(t)) .
$$

Moreover, for every $t \in[0, T]$

$$
\Gamma^{k}(t) \cup \partial_{N} \Omega \rightarrow \Gamma(t) \cup \partial_{N} \Omega \quad \text { in the sense of } \sigma^{p} \text {-convergence on } \bar{\Omega} \text {, }
$$

and for a subsequence $\left(k_{t}\right)$ (possibly depending on $t$ )

$$
u^{k_{t}}(t) \rightarrow u(t) \quad \text { weakly in } S B V^{p}(\Omega) .
$$

\section{Regular quasi-static evolutions in the two-dimensional anti-plane setting}

In this section we show that in dimension two, and under suitable assumptions on the reference configuration, the boundary displacements and the bulk energy density, there exists a quasi-static evolution whose associated crack is, at any time, 
topologically closed. As a consequence, also the associated displacements enjoy more regularity, they are Sobolev and Hölder continuous outside the crack. We will speak of regular quasi-static evolutions.

Let us consider the case $N=2$. Assume that the reference configuration $\Omega \subseteq \mathbb{R}^{2}$ is such that

$$
\begin{cases}\Omega \subseteq \mathbb{R}^{2} & \text { is open, bounded and with Lipschitz boundary, } \\ \partial_{D} \Omega \subseteq \partial \Omega & \text { is open in the relative topology and of class } \mathcal{C}^{1}, \\ \partial_{N} \Omega:=\partial \Omega \backslash \partial_{D} \Omega . & \end{cases}
$$

We assume that the elastic energy density $W: \mathbb{R}^{2} \rightarrow \mathbb{R}$ and the surface energy density $\kappa: \mathbb{R}^{2} \rightarrow \mathbb{R}$ are such that

$W$ is of class $\mathcal{C}^{1}$ and satisfies $\left(\mathrm{H}_{1}\right)-\left(\mathrm{H}_{3}\right)$ of Section 3 , and $\kappa$ satisfies (3.2).

Note that in view of Remark $3.1, W$ is uniformly convex on $\mathbb{R}^{2}$.

Let us consider a prescribed boundary displacement $g$ such that

$$
g \in A C\left(0, T ; W^{1, \infty}(\Omega)\right) .
$$

Finally let us assume that the globally stable initial configuration $\left(u_{0}, \Gamma_{0}\right) \in \mathcal{A}(g(0))$ is such that $\Gamma_{0} \subseteq \Omega \cup \partial_{D} \Omega$ is closed in the relative topology, and for every $\eta>0$ there exist $\vartheta_{0}>0$ and $\rho_{0}>0$ such that for every $x \in \Gamma_{0}$ and $\rho \leq \rho_{0}$ with $\operatorname{dist}\left(B_{\rho}(x), \partial_{N} \Omega\right)>\eta$

$$
\mathcal{H}^{1}\left(\Gamma_{0} \cap B_{\rho}(x)\right) \geq \vartheta_{0} \rho .
$$

Remark 5.1. Note that the family of all admissible initial configurations is not empty. Indeed, let $\Omega_{0} \subseteq \mathbb{R}^{2}$ be a bounded open set such that $\bar{\Omega} \subseteq \Omega_{0}$, and set $\Omega^{\prime}:=\Omega_{0} \backslash \partial_{N} \Omega$. Let us extend $g(0)$ to a function in $W^{1, \infty}\left(\Omega_{0}\right)$, and let $u_{0} \in S B V^{p}\left(\Omega^{\prime}\right)$ be a solution of

$\min \left\{\int_{\Omega^{\prime}} W(\nabla v) d x+\int_{S_{v}} \kappa\left(v_{v}\right) d \mathcal{H}^{N-1}: v \in S B V^{p}\left(\Omega^{\prime}\right), v=g(0)\right.$ a.e. in $\left.\Omega^{\prime} \backslash \bar{\Omega}\right\}$.

From Proposition 3.11, $u_{0}-g(0)$ is a quasi-minimizer of the functional (3.3) with $A=\Omega^{\prime}$. By Theorem 3.4 and Remark 3.5, the set $\Gamma_{0}:=S_{u_{0}} \widetilde{\subset} \Omega \cup \partial_{D} \Omega$ is essentially relatively closed in $\Omega \cup \partial_{D} \Omega$ and satisfies (5.4) for suitable $\vartheta_{0}, \rho_{0}>0$.

The main result of the paper is the following:

Theorem 5.2 (Existence of regular quasi-static evolutions). Assume (5.1), (5.2), (5.3) and (5.4). Then there exists a quasi-static evolution $[0, T] \ni t \mapsto(u(t), \Gamma(t))$ relative to the boundary displacement $g$, with $(u(0), \Gamma(0))=\left(u_{0}, \Gamma_{0}\right)$, such that for every $t \in[0, T]$

$$
\Gamma(t) \subseteq \Omega \cup \partial_{D} \Omega \text { is closed in the relative topology, }
$$

and, for some $\alpha \in(0,1)$ independent of $t$,

$$
u(t) \in W^{1, p}(\Omega \backslash \Gamma(t)) \cap L^{\infty}(\Omega) \cap \mathcal{C}_{\mathrm{loc}}^{0, \alpha}\left(\left(\Omega \cup \partial_{D} \Omega\right) \backslash \Gamma(t)\right) .
$$


In order to prove existence of regular quasi-static evolutions, we study more in details the discrete in time evolutions which are used to establish the existence of quasi-static evolutions. We will prove that incremental configurations enjoy additional regularity properties (Proposition 5.3), and that, thanks to a uniform lower bound for the density of the cracks (Proposition 5.5), this regularity is transferred to the associated quasi-static evolutions as the time step discretization vanishes (Theorem 5.7).

As in the previous section, let us take a discretization $\left\{t_{i}^{k}\right\}_{0 \leq i \leq n(k)}$ of the time interval $[0, T]$ satisfying (4.4) and (4.5), and set $g_{i}^{k}:=g\left(t_{i}^{k}\right)$.

In order to deal with the Dirichlet boundary condition, let us introduce a bounded open set $\Omega_{0} \subseteq \mathbb{R}^{2}$ such that $\bar{\Omega} \subseteq \Omega_{0}$ and $\operatorname{diam}\left(\Omega_{0}\right) \leq 2 \operatorname{diam}(\Omega)$. By using an extension operator, we may assume that

$$
g \in A C\left(0, T ; W^{1, \infty}\left(\Omega_{0}\right)\right) .
$$

Given $(u, \Gamma) \in \mathcal{A}(g(t))$, we extend $u$ to $\Omega_{0}$ by setting $u=g(t)$ on $\Omega_{0} \backslash \Omega$ obtaining a function in $S B V^{p}\left(\Omega_{0}\right)$. Note that we may have created a jump on the Neumann part $\partial_{N} \Omega$ with

$$
S_{u} \widetilde{\subset} \Gamma \cup \partial_{N} \Omega,
$$

but this additional jump is not counted in the energy (4.1). As a consequence, $\mathcal{A}(g(t))$ may be described as

$\mathcal{A}(g(t))=\left\{(u, \Gamma): u \in S B V^{p}\left(\Omega_{0}\right), \Gamma \widetilde{\subset} \bar{\Omega}, S_{u} \widetilde{\subset} \partial_{N} \Omega \cup \Gamma, u=g(t)\right.$ a.e. in $\left.\Omega_{0} \backslash \Omega\right\}$.

The following result holds:

Proposition 5.3 (Regular incremental configurations). Assume (5.1), (5.2), (5.3) and (5.4). There exist incremental configurations $\left(u_{i}^{k}, \Gamma_{i}^{k}\right) \in \mathcal{A}\left(g_{i}^{k}\right)$ with $\left(u_{0}^{k}, \Gamma_{0}^{k}\right)=$ $\left(u_{0}, \Gamma_{0}\right)$, such that for every $k \in \mathbb{N}$ and $i \in\{0, \ldots, n(k)\}$,

$$
\begin{gathered}
\Gamma_{i}^{k}=\Gamma_{i-1}^{k} \cup\left(\overline{S_{u_{i}^{k}}} \backslash \partial_{N} \Omega\right), \\
\Gamma_{i}^{k} \subseteq \Omega \cup \partial_{D} \Omega \text { is closed in the relative topology, }
\end{gathered}
$$

and for some $\alpha \in(0,1)$ independent of $k$ and $i$

$$
u_{i}^{k} \in W^{1, p}\left(\Omega \backslash \Gamma_{i}^{k}\right) \cap L^{\infty}(\Omega) \cap \mathcal{C}_{\text {loc }}^{0, \alpha}\left(\left(\Omega \cup \partial_{D} \Omega\right) \backslash \Gamma_{i}^{k}\right) .
$$

Finally for every $\eta>0$, there exist $\vartheta_{0}>0$ and $\rho_{0}>0$ (independent of $k$ and $i$ ) such that

$$
\mathcal{H}^{1}\left(\Gamma_{i}^{k} \cap B_{\rho}(x)\right) \geq \vartheta_{0} \rho
$$

for every ball $B_{\rho}(x) \subseteq \Omega_{0} \backslash\left(\partial_{N} \Omega \cup \Gamma_{i-1}^{k}\right)$ with center $x \in \Gamma_{i}^{k}$, radius $\rho \leq \rho_{0}$, and such that $\operatorname{dist}\left(B_{\rho}(x), \partial_{N} \Omega\right)>\eta$. 
Proof. Assuming having constructed $\left(u_{i-1}^{k}, \Gamma_{i-1}^{k}\right) \in \mathcal{A}\left(g_{i-1}^{k}\right)$ at time $t_{i-1}^{k}$ for $i \geq$ 1 , with $\Gamma_{i-1}^{k} \subseteq \Omega \cup \partial_{D} \Omega$ closed in the relative topology of $\Omega \cup \partial_{D} \Omega$, let $u_{i}^{k} \in$ $S B V^{p}\left(\Omega_{0}\right)$ be a solution of the following problem

$\min \left\{\int_{\Omega} W(\nabla v) d x+\int_{S_{v} \backslash\left(\Gamma_{i-1}^{k} \cup \partial_{N} \Omega\right)} \kappa\left(v_{v}\right) d \mathcal{H}^{1}: v \in S B V^{p}\left(\Omega_{0}\right), v=g_{i}^{k}\right.$ a.e. in $\left.\Omega_{0} \backslash \Omega\right\}$.

The existence of $u_{i}^{k}$ is guaranteed by Ambrosio's theorem (Theorem 2.1) and the lower semicontinuity property (2.1) since $W(0)=0$, and by truncation we may assume that $\|v\|_{\infty} \leq\left\|g_{i}^{k}\right\|_{\infty}$. Clearly $S_{u_{i}^{k}} \subseteq \bar{\Omega}$ as $g_{i}^{k}$ is locally Lipschitz on $\Omega_{0} \backslash \bar{\Omega}$.

Let us consider the open sets

$$
\Omega^{\prime}:=\Omega_{0} \backslash \partial_{N} \Omega \quad \text { and } \quad A:=\Omega_{0} \backslash\left(\partial_{N} \Omega \cup \Gamma_{i-1}^{k}\right) .
$$

It is readily seen that the restriction of $u_{i}^{k}$ to $A$ is a solution of (3.6) with $g=$ $g_{i}^{k}$ (compare firstly with bounded functions in $S B V^{p}(A)$ which can be seen as functions in $S B V^{p}\left(\Omega_{0}\right)$ since $\mathcal{H}^{1}\left(\partial_{N} \Omega \cup \Gamma_{i-1}^{k}\right)<+\infty$, and then use a truncation argument). By Proposition 3.11, the function

$$
u_{i}^{k}-g_{i}^{k} \in S B V^{p}(A)
$$

is a quasi-minimizer of (3.3) with constants $s=1 / p$ and $\omega=\omega_{i}^{k}$. Note that $\omega_{i}^{k}$ only depends on $N, p, L, \beta,\left\|\nabla g_{i}^{k}\right\|_{\infty}, \operatorname{diam}(\Omega)$, and the Lipschitz constant of $\partial \Omega$. However, since $\sup _{t \in[0, T]}\|\nabla g(t)\|_{\infty}<+\infty$, we infer that $\omega_{i}^{k} \leq \omega$ for some $\omega$ independent of $i$ and $k$.

By Theorem 3.4 and Remark 3.5, since clearly $S_{u_{i}^{k}}=S_{u_{i}^{k}-g_{i}^{k}}$, the set $S_{u_{i}^{k}}$ is essentially closed in $A$, and for every $\eta>0$ there exist $\vartheta_{0}>0$ and $\rho_{0}>0$ (depending only on $N, p, L, \mu, \beta, g$, $\operatorname{diam}(\Omega)$, the Lipschitz constant of $\partial \Omega$ and $\eta$ ) such that

$$
\mathcal{H}^{1}\left(S_{u_{i}^{k}} \cap B_{\rho}(x)\right) \geq \vartheta_{0} \rho
$$

for all balls $B_{\rho}(x) \subseteq A$ with center $x \in \overline{S_{u_{i}^{k}}}$, radius $\rho \leq \rho_{0}$, and such that $\operatorname{dist}\left(B_{\rho}(x), \partial_{N} \Omega\right)>\eta$. Note that the constants $\left(\rho_{0}, \vartheta_{0}\right)$ given by Theorem 3.4 can be taken to be equal to those of (5.4) involving $\Gamma_{0}$.

By considering the restriction of $u_{i}^{k}$ to $\Omega$ and setting

$$
\Gamma_{i}^{k}:=\Gamma_{i-1}^{k} \cup\left(\overline{S_{u_{i}^{k}}} \backslash \partial_{N} \Omega\right)
$$

we obtain that $\left(u_{i}^{k}, \Gamma_{i}^{k}\right) \in \mathcal{A}\left(g_{i}^{k}\right)$ with $\Gamma_{i}^{k} \subseteq \Omega \cup \partial_{D} \Omega$ closed in the relative topology of $\Omega \cup \partial_{D} \Omega$, and $u_{i}^{k} \in W^{1, p}\left(\Omega \backslash \Gamma_{i}^{k}\right) \cap L^{\infty}(\Omega)$. It is easily seen that $\left(u_{i}^{k}, \Gamma_{i}^{k}\right)$ are incremental configurations, i.e., they are solutions of (4.6) satisfying the $L^{\infty}$-bound (4.7). By the global stability of $\left(u_{i}^{k}, \Gamma_{i}^{k}\right)$, we deduce that

$$
\int_{\Omega \backslash \Gamma_{i}^{k}} W\left(\nabla u_{i}^{k}\right) d x \leq \int_{\Omega \backslash \Gamma_{i}^{k}} W(\nabla v) d x
$$


for any $v \in W^{1, p}\left(\Omega \backslash \Gamma_{i}^{k}\right)$ such that $v=g_{i}^{k} \mathcal{H}^{1}$-a.e. on $\partial_{D} \Omega \backslash \Gamma_{i}^{k}$ (in the sense of traces).

Since

$$
\sup _{k, i}\left(\left\|u_{i}^{k}\right\|_{\infty}+\left\|\nabla g_{i}^{k}\right\|_{\infty}\right)<+\infty,
$$

according to [18, formulas (7.45) and (7.54)], we infer that there exists $\alpha \in(0,1)$ independent of $k$ and $i$ such that

$$
u_{i}^{k} \in \mathcal{C}_{\mathrm{loc}}^{0, \alpha}\left(\left(\Omega \cup \partial_{D} \Omega\right) \backslash \Gamma_{i}^{k}\right) .
$$

Finally (5.5) follows from (5.6), so that the proof is concluded.

Remark 5.4. Assume that $A \subseteq \Omega$ is open and such that $\bar{A} \cap\left(\partial_{N} \Omega \cup \Gamma_{i}^{k}\right)=\emptyset$ for every $k, i$. In view of (5.7) and according to [18, formulas (7.45) and (7.54)], we infer that

$$
\sup _{k, i}\left\|u_{i}^{k}\right\|_{\mathcal{C}^{0, \alpha}(\bar{A})}<+\infty .
$$

Moving to the associated discrete in time evolutions, we can formulate the following uniform density lower bound for the cracks.

Proposition 5.5 (Uniform in time density lower bound). Assume (5.1), (5.2), (5.3) and (5.4), and let $[0, T] \ni t \mapsto\left(u^{k}(t), \Gamma^{k}(t)\right)$ be the discrete in time evolution associated to the incremental configurations given by Proposition 5.3.

For every $\eta>0$, there exist $\vartheta_{0}>0$ and $\rho_{0}>0$ (independent of $k$ and $t$ ) such that for all $t \in[0, T]$,

$$
\mathcal{H}^{1}\left(\Gamma^{k}(t) \cap B_{\rho}(x)\right) \geq \vartheta_{0} \rho
$$

for any ball $B_{\rho}(x) \subseteq \Omega_{0} \backslash \partial_{N} \Omega$ of center $x \in \Gamma^{k}(t)$, radius $\rho \leq \rho_{0}$, and such that $\operatorname{dist}\left(B_{\rho}(x), \partial_{N} \Omega\right)>\eta$.

Proof. The proof is based on a simple geometrical argument which works in dimension two. Let $\vartheta_{0}$ and $\rho_{0}$ be given by Proposition 5.3. It suffices to prove that (5.8) holds for $\Gamma_{i}^{k}$ for every $k \in \mathbb{N}$ and $i \in\{0, \ldots, n(k)\}$. We proceed by induction on $i$.

For $i=0$, the density lower bound holds by assumption (5.4) since $\Gamma_{0}^{k}=\Gamma_{0}$ (and $\vartheta_{0}, \rho_{0}$ can be considered compatible with the constants in (5.4)). Assume that (5.8) holds for $\Gamma_{i-1}^{k}$, and let us prove it for $\Gamma_{i}^{k}=\Gamma_{i-1}^{k} \cup\left(\overline{S_{u_{i}^{k}}} \backslash \partial_{N} \Omega\right)$. Let us consider

$$
x \in \overline{S_{u_{i}^{k}}} \backslash\left(\Gamma_{i-1}^{k} \cup \partial_{N} \Omega\right),
$$

and let $\rho \leq \rho_{0}$ be such that $B_{\rho}(x) \subseteq \Omega_{0} \backslash \partial_{N} \Omega$ and $\operatorname{dist}\left(B_{\rho}(x), \partial_{N} \Omega\right)>\eta$. Define $\delta_{0}:=\operatorname{dist}\left(x, \Gamma_{i-1}^{k} \cup \partial_{N} \Omega\right)>0$ and consider $y \in \Gamma_{i-1}^{k} \cup \partial_{N} \Omega$ such that $\delta_{0}=|x-y|$.

If $\rho \leq \delta_{0}$, the desired inequality follows by (5.5) since in that case $B_{\rho}(x) \subseteq$ $\Omega_{0} \backslash\left(\partial_{N} \Omega \cup \Gamma_{i-1}^{k}\right)$. 


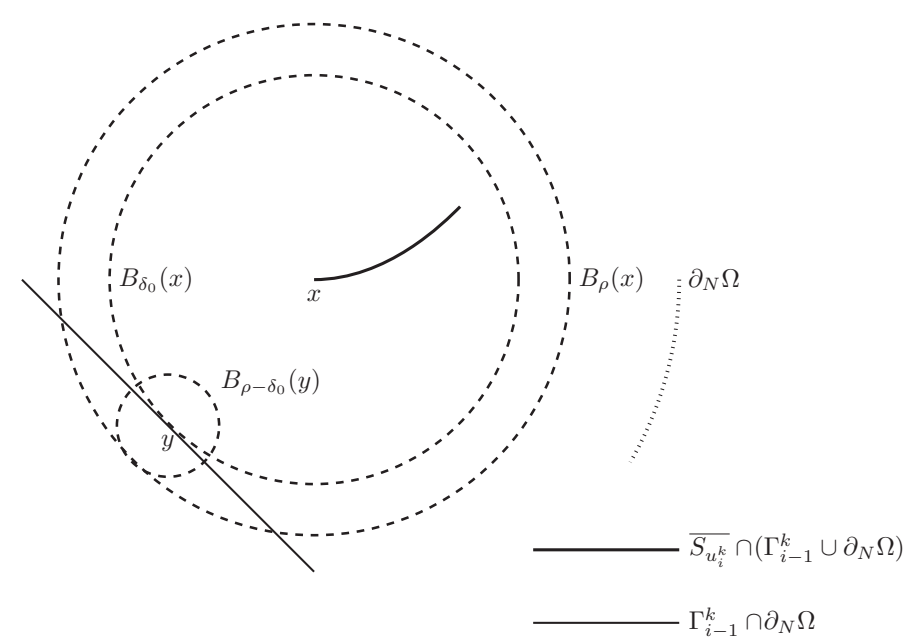

Figure 5.1. The $2 D$ geometrical argument.

If $\rho>\delta_{0}$, then necessarily $y \in \Gamma_{i-1}^{k} \backslash \partial_{N} \Omega\left(\right.$ since $B_{\rho}(x)$ does not intersect $\left.\partial_{N} \Omega\right)$. Then in view of the inductive assumption on $\Gamma_{i-1}^{k}$ and of (5.5), we infer that (see Figure 5.1)

$$
\begin{aligned}
\mathcal{H}^{1}\left(\Gamma_{i}^{k} \cap B_{\rho}(x)\right) & \geq \mathcal{H}^{1}\left(\Gamma_{i}^{k} \cap B_{\delta_{0}}(x)\right)+\mathcal{H}^{1}\left(\Gamma_{i-1}^{k} \cap B_{\rho-\delta_{0}}(y)\right) \\
& \geq \vartheta_{0} \delta_{0}+\vartheta_{0}\left(\rho-\delta_{0}\right)=\vartheta_{0} \rho .
\end{aligned}
$$

Remark 5.6. Following the previous estimates in the case $N \geq 3$, we would end up with the inequality

$$
\mathcal{H}^{N-1}\left(\Gamma_{i}^{k} \cap B_{\rho}(x)\right) \geq \varepsilon^{i} \vartheta_{0} \rho^{N-1}
$$

with $\varepsilon \in(0,1)$, which degenerates as $k \rightarrow+\infty$.

We are now in position to prove Theorem 5.2. It is a consequence of the following result.

Theorem 5.7 (Convergence to a regular quasi-static evolution). Assume (5.1), (5.2), (5.3), (5.4), and let $[0, T] \ni t \mapsto\left(u^{k}(t), \Gamma^{k}(t)\right)$ be the discrete in time evolution given by Proposition 5.5 .

There exists a (not relabeled) subsequence (independent of $t)$ of $\left(u^{k}(t), \Gamma^{k}(t)\right)$ and a quasi-static evolution $[0, T] \ni t \mapsto(u(t), \Gamma(t))$ relative to the boundary displacement $g$, with $(u(0), \Gamma(0))=\left(u_{0}, \Gamma_{0}\right)$, and such that the following properties are satisfied.

(a) For every $t \in[0, T]$

$$
\Gamma(t) \subseteq \Omega \cup \partial_{D} \Omega \text { is closed in relative topology of } \Omega \cup \partial_{D} \Omega
$$


and, for some $\alpha \in(0,1)$ independent of $t$,

$$
u(t) \in W^{1, p}(\Omega \backslash \Gamma(t)) \cap L^{\infty}(\Omega) \cap \mathcal{C}_{\mathrm{loc}}^{0, \alpha}\left(\left(\Omega \cup \partial_{D} \Omega\right) \backslash \Gamma(t)\right) .
$$

(b) For every $t \in[0, T]$

$$
\Gamma^{k}(t) \cup \partial_{N} \Omega \rightarrow \Gamma(t) \cup \partial_{N} \Omega \quad \text { in the Hausdorff metric, }
$$

and

$$
\int_{\Gamma^{k}(t)} \kappa\left(v_{\Gamma^{k}(t)}\right) d \mathcal{H}^{1} \rightarrow \int_{\Gamma(t)} \kappa\left(v_{\Gamma(t)}\right) d \mathcal{H}^{1}
$$

(c) For every $t \in[0, T]$

$$
\nabla u^{k}(t) \rightarrow \nabla u(t) \quad \text { strongly in } L^{p}\left(\Omega ; \mathbb{R}^{2}\right)
$$

and

$$
\int_{\Omega} W\left(\nabla u^{k}(t)\right) d x \rightarrow \int_{\Omega} W(\nabla u(t)) d x .
$$

Finally, for every $A \subseteq \Omega$ open, connected, with Lipschitz boundary, such that $\bar{A} \cap\left(\partial_{N} \Omega \cup \Gamma(t)\right)=\emptyset$ and $\mathcal{H}^{1}\left(\partial A \cap\left(\partial_{D} \Omega \backslash \Gamma(t)\right)\right)>0$, then $\bar{A} \cap\left(\partial_{N} \Omega \cup\right.$ $\left.\Gamma^{k}(t)\right)=\emptyset$ for $k$ large enough and

$$
u^{k}(t) \rightarrow u(t) \text { uniformly in } \bar{A} .
$$

Proof. Let us consider the quasi-static evolution $[0, T] \ni t \mapsto(u(t), \Gamma(t))$ associated to a subsequence of $t \mapsto\left(u^{k}(t), \Gamma^{k}(t)\right)$ according to Theorem 4.4. We know that (5.12) holds true.

Concerning the cracks, we have $\Gamma^{k}(t), \Gamma(t) \widetilde{\subset} \Omega \cup \partial_{D} \Omega$,

$$
\Gamma^{k}(t) \cup \partial_{N} \Omega \rightarrow \Gamma(t) \cup \partial_{N} \Omega \quad \text { in the sense of } \sigma^{p} \text {-convergence on } \bar{\Omega},
$$

and (5.10) is a consequence of (4.9).

Since the sets $\Gamma^{k}(t) \cup \partial_{N} \Omega$ are closed in $\bar{\Omega}$ and increasing in time, by [11, Theorem 6.3], up to a further subsequence independent of $t$, we can assume that there exists an increasing map $t \mapsto K_{N}(t)$, with $K_{N}(t) \subseteq \bar{\Omega}$ compact and such that for every $t \in[0, T]$

$$
\Gamma^{k}(t) \cup \partial_{N} \Omega \rightarrow K_{N}(t) \quad \text { in the Hausdorff metric. }
$$

Let us set

$$
K(t):=K_{N}(t) \backslash \partial_{N} \Omega
$$

so that $K(t) \subseteq \Omega \cup \partial_{D} \Omega$ is closed in the relative topology of $\Omega \cup \partial_{D} \Omega$.

Concerning the displacements, let us prove (5.11). This improvement for the convergence of $\nabla u^{k}(t)$ to $\nabla u(t)$ (strong convergence and no need of subsequences depending on $t)$ is due to the uniform convexity properties of $W$. Let $\left(\nabla u^{k_{j}}(t)\right)$ be 
a subsequence of $\left(\nabla u^{k}(t)\right)$. There exists a further time-dependent subsequence (not relabeled) such that

$$
u^{k_{j}}(t) \rightarrow v \quad \text { weakly in } S B V^{p}(\Omega)
$$

for some $v \in S B V^{p}(\Omega)$ with $(v, \Gamma(t)) \in \mathcal{A}(g(t))$ (admissibility is a consequence of the $\sigma^{p}$-convergence of the cracks). In view of the global stability of $\left(u^{k}(t), \Gamma^{k}(t)\right)$, the Jump Transfer Lemma [8, Theorem 5.1] immediately entails that

$$
\int_{\Omega} W(\nabla v) d x \leq \int_{\Omega} W(\nabla z) d x
$$

for any $z \in S B V^{p}(\Omega)$ such that $(z, \Gamma(t)) \in \mathcal{A}(g(t))$. Note that $u(t)$ also enjoys the same property. Since the set $\left\{\nabla z \in L^{p}\left(\Omega ; \mathbb{R}^{2}\right):(z, \Gamma(t)) \in \mathcal{A}(g(t))\right\}$ is convex and $W$ is strictly uniformly convex (see Remark 3.1), i.e. $\xi \mapsto W(\xi)-c|\xi|^{p}$ is convex for some $c>0$, we deduce that $\nabla v=\nabla u(t)$. As a consequence, along the entire sequence

$$
\nabla u^{k}(t) \rightarrow \nabla u(t) \quad \text { weakly in } L^{p}\left(\Omega ; \mathbb{R}^{2}\right) .
$$

In view of (5.12), and since $W$ is strictly uniformly convex, the convergence is readily seen to be strong, so that (5.11) follows.

In order to complete the proof, we now proceed in several steps.

Step 1: Localizing the convergence of the crack energy. Let us prove that the convergence of the surface energy (5.10) can actually be localized on almost every balls, i.e., for every $x \in \Omega_{0} \backslash \partial_{N} \Omega$ and a.e. $\rho>0$ with $B_{\rho}(x) \subseteq \Omega_{0} \backslash \partial_{N} \Omega$, then

$$
\lim _{k \rightarrow+\infty} \int_{\Gamma^{k}(t) \cap B_{\rho}(x)} \kappa\left(v_{\Gamma^{k}(t)}\right) d \mathcal{H}^{1}=\int_{\Gamma(t) \cap B_{\rho}(x)} \kappa\left(v_{\Gamma(t)}\right) d \mathcal{H}^{1}
$$

By the lower semicontinuity property of the $\sigma^{p}$-convergence (see Proposition 2.4), we have

$$
\begin{aligned}
& \liminf _{k \rightarrow+\infty} \int_{\Gamma^{k}(t) \cap B_{\rho}(x)} \kappa\left(v_{\Gamma^{k}(t)}\right) d \mathcal{H}^{1}=\liminf _{k \rightarrow+\infty} \mathcal{K}\left(\left(\Gamma^{k}(t) \cup \partial_{N} \Omega\right) \cap B_{\rho}(x)\right) \\
& \geq \mathcal{K}\left(\left(\Gamma(t) \cup \partial_{N} \Omega\right) \cap B_{\rho}(x)\right)=\int_{\Gamma(t) \cap B_{\rho}(x)} \kappa\left(v_{\Gamma(t)}\right) d \mathcal{H}^{1}
\end{aligned}
$$

hence it remains to prove that

$$
\limsup _{k \rightarrow+\infty} \int_{\Gamma^{k}(t) \cap B_{\rho}(x)} \kappa\left(v_{\Gamma^{k}(t)}\right) d \mathcal{H}^{1} \leq \int_{\Gamma(t) \cap B_{\rho}(x)} \kappa\left(v_{\Gamma(t)}\right) d \mathcal{H}^{1} .
$$

First of all, we notice that there exists a countable set $S \subset\left(0, \operatorname{dist}\left(x, \partial\left(\Omega_{0} \backslash \partial_{N} \Omega\right)\right)\right.$ such that for all $\rho \notin S$

$$
\mathcal{H}^{1}\left(\Gamma(t) \cap \partial B_{\rho}(x)\right)=0
$$


Select such a radius $\rho \notin S$, and assume that (5.16) does not hold, then

$$
\limsup _{k \rightarrow+\infty} \int_{\Gamma^{k}(t) \cap B_{\rho}(x)} \kappa\left(v_{\Gamma^{k}(t)}\right) d \mathcal{H}^{1}>\int_{\Gamma(t) \cap B_{\rho}(x)} \kappa\left(v_{\Gamma(t)}\right) d \mathcal{H}^{1} .
$$

On the other hand, using again Proposition 2.4, we have that

$$
\begin{aligned}
\liminf _{k \rightarrow+\infty} \int_{\Gamma^{k}(t) \backslash \overline{B_{\rho}(x)}} \kappa\left(v_{\Gamma^{k}(t)}\right) d \mathcal{H}^{1}=\liminf _{k \rightarrow+\infty} \mathcal{K}\left(\left(\Gamma^{k}(t) \cup \partial_{N} \Omega\right) \backslash \overline{B_{\rho}(x)}\right) \\
\geq \mathcal{K}\left(\left(\Gamma(t) \cup \partial_{N} \Omega\right) \backslash \overline{B_{\rho}(x)}\right)=\int_{\Gamma(t) \backslash \overline{B_{\rho}(x)}} \kappa\left(v_{\Gamma(t)}\right) d \mathcal{H}^{1} .
\end{aligned}
$$

Consequently, in view of (5.17) and (5.18), we deduce that

$$
\limsup _{k \rightarrow+\infty} \int_{\Gamma^{k}(t)} \kappa\left(v_{\Gamma^{k}(t)}\right) d \mathcal{H}^{1}>\int_{\Gamma(t)} \kappa\left(v_{\Gamma(t)}\right) d \mathcal{H}^{1}
$$

which is against (5.10). We conclude that (5.15) holds.

Step 2: Hausdorff convergence for the cracks. We are now in position to prove that $\Gamma(t) \cong K(t)$, where $K(t)$ is given in (5.14), so that we can assume $\Gamma(t) \subseteq$ $\Omega \cup \partial_{D} \Omega$ closed in the relative topology of $\Omega \cup \partial_{D} \Omega$ and

$$
\Gamma^{k}(t) \cup \partial_{N} \Omega \rightarrow \Gamma(t) \cup \partial_{N} \Omega \quad \text { in the Hausdorff metric. }
$$

Hence item (b) and the first part of item (a) hold true.

By definition of $\sigma^{p}$ and Hausdorff convergences, we always have that $\Gamma(t) \widetilde{C}$ $K(t)$. Assume by contradiction $\mathcal{H}^{1}(K(t) \backslash \Gamma(t))>0$. Then by [3, Section 2.9] there exists $x \in K(t) \backslash \Gamma(t)$ such that

$$
\lim _{\rho \rightarrow 0} \frac{\mathcal{H}^{1}\left(\Gamma(t) \cap B_{\rho}(x)\right)}{\rho}=0 .
$$

Let us fix $\eta>0$ such that

$$
2 \eta<\operatorname{dist}\left(x, \partial_{N} \Omega\right) .
$$

There exists $\rho<\min \left\{\rho_{0}, \eta\right\}$ such that $B_{\rho}(x) \subseteq \Omega_{0} \backslash \partial_{N} \Omega$ and

$$
\mathcal{H}^{1}\left(\Gamma(t) \cap B_{\rho}(x)\right)<\vartheta_{0} \beta^{-2} \rho,
$$

where $\rho_{0}$ and $\vartheta_{0}$ are the constants appearing in Proposition 5.5 associated to $\eta$, while $\beta$ appears in the coercivity estimate for the surface energy density $\kappa$ in (3.2). By (5.15), there exists $\rho^{\prime}<\rho$ (which can be chosen arbitrarily close to $\rho$ ) such that

$$
\lim _{k \rightarrow+\infty} \int_{\Gamma^{k}(t) \cap B_{\rho^{\prime}}(x)} \kappa\left(v_{\Gamma^{k}(t)}\right) d \mathcal{H}^{1}=\int_{\Gamma(t) \cap B_{\rho^{\prime}}(x)} \kappa\left(v_{\Gamma(t)}\right) d \mathcal{H}^{1} .
$$


Since $\Gamma^{k}(t) \cup \partial_{N} \Omega$ converges to $K(t) \cup \partial_{N} \Omega$ in the Hausdorff metric, and $x \in$ $K(t)$, it is possible to find a sequence of points $x_{k} \in \Gamma^{k}(t) \cup \partial_{N} \Omega$ with $x_{k} \rightarrow$ $x$. Being $\operatorname{dist}\left(B_{\rho}(x), \partial_{N} \Omega\right)>\eta$, then $x_{k} \in \Gamma^{k}(t)$ for $k$ large enough. Hence if $\rho^{\prime \prime}<\rho^{\prime}$, then for $k$ large enough one has $B_{\rho^{\prime \prime}}\left(x_{k}\right) \subset B_{\rho^{\prime}}(x) \subseteq \Omega_{0} \backslash \partial_{N} \Omega$ and $\operatorname{dist}\left(B_{\rho^{\prime \prime}}\left(x_{k}\right), \partial_{N} \Omega\right)>\eta$, and thus by (5.20) and (5.8)

$$
\begin{aligned}
\beta \mathcal{H}^{1}\left(\Gamma(t) \cap B_{\rho^{\prime}}(x)\right) & \geq \int_{\Gamma(t) \cap B_{\rho^{\prime}}(x)} \kappa\left(v_{\Gamma(t)}\right) d \mathcal{H}^{1} \\
& =\lim _{k \rightarrow+\infty} \int_{\Gamma^{k}(t) \cap B_{\rho^{\prime}}(x)} \kappa\left(v_{\Gamma^{k}(t)}\right) d \mathcal{H}^{1} \\
& \geq \limsup _{k \rightarrow+\infty} \int_{\Gamma^{k}(t) \cap B_{\rho^{\prime \prime}}\left(x_{k}\right)} \kappa\left(v_{\Gamma^{k}(t)}\right) d \mathcal{H}^{1} \\
& \geq \beta^{-1} \limsup _{k \rightarrow+\infty} \mathcal{H}^{1}\left(\Gamma^{k}(t) \cap B_{\rho^{\prime \prime}}\left(x_{k}\right)\right) \geq \vartheta_{0} \beta^{-1} \rho^{\prime \prime} .
\end{aligned}
$$

Letting first $\rho^{\prime \prime} \nearrow \rho^{\prime}$, and then $\rho^{\prime} \nearrow \rho$, we deduce that

$$
\mathcal{H}^{1}\left(\Gamma(t) \cap B_{\rho}(x)\right) \geq \vartheta_{0} \beta^{-2} \rho,
$$

which is in contradiction with (5.19).

Step 3: Regularity of the displacements. Let us complete the proof of item (a).

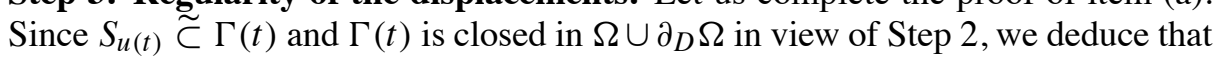
(recall that $\left.\|u(t)\|_{\infty} \leq\|g(t)\|_{\infty}\right)$

$$
u(t) \in W^{1, p}(\Omega \backslash \Gamma(t)) \cap L^{\infty}(\Omega) .
$$

Next using the global stability of $(u(t), \Gamma(t))$, we deduce that

$$
\int_{\Omega \backslash \Gamma(t)} W(\nabla u(t)) d x \leq \int_{\Omega \backslash \Gamma(t)} W(\nabla v) d x
$$

for any $v \in W^{1, p}(\Omega \backslash \Gamma(t))$ such that $v=g(t) \mathcal{H}^{1}$-a.e. on $\partial_{D} \Omega \backslash \Gamma(t)$ (in the sense of traces).

Since

$$
\sup _{t \in[0, T]}\left(\|u(t)\|_{\infty}+\|\nabla g(t)\|_{\infty}\right)<+\infty,
$$

by [18, Theorem 7.8], there exists $\alpha>0$ (independent of $t$ ) such that

$$
u(t) \in \mathcal{C}_{\mathrm{loc}}^{0, \alpha}\left(\left(\Omega \cup \partial_{D} \Omega\right) \backslash \Gamma(t)\right)
$$

Step 4: Uniform convergence for the displacements. Let us finally prove (5.13). Let $A \subseteq \Omega$ be open, connected, with Lipschitz boundary, such that $\bar{A} \cap\left(\partial_{N} \Omega \cup\right.$ $\Gamma(t))=\varnothing$ and $\mathcal{H}^{1}\left(\partial A \cap\left(\partial_{D} \Omega \backslash \Gamma(t)\right)\right)>0$. Consider $k$ large enough so that 
$\bar{A} \cap\left(\partial_{N} \Omega \cup \Gamma^{k}(t)\right)=\emptyset$ : this is possible in view of the Hausdorff convergence in (5.9). In particular $u^{k}(t) \in W^{1, p}(A)$ for $k$ large enough, and

$$
u^{k_{j}}(t) \rightarrow v \quad \text { weakly in } W^{1, p}(A)
$$

for some subsequence $\left(k_{j}\right)$. Clearly $\nabla v=\nabla u(t)$ in view of (5.11). Since $g^{k}(t) \rightarrow$ $g(t)$ uniformly on $\bar{A} \cap\left(\partial_{D} \Omega \backslash \Gamma(t)\right)$, we infer that $v=g(t) \mathcal{H}^{1}$-a.e. on $\partial A \cap\left(\partial_{D} \Omega \backslash\right.$ $\Gamma(t))$. Hence we deduce $v=u(t)$, so that without passing to subsequences

$$
u^{k}(t) \rightarrow u(t) \quad \text { weakly in } W^{1, p}(A) .
$$

The convergence is indeed uniform on $\bar{A}$ : it is a consequence of Ascoli-Arzelà theorem since in view of Remark 5.4

$$
\sup _{k \in \mathbb{N}}\left\|u^{k}(t)\right\|_{\mathcal{C}^{0, \alpha}(\bar{A})}<+\infty .
$$

Remark 5.8. Note that the main place where we used our version of the density lower bound given in Proposition 5.5 on balls centered in $\Omega$ but touching $\partial_{D} \Omega$ is formula (5.21). Indeed, in the case $\Gamma^{k}(t) \subseteq \Omega$ but with $\Gamma(t) \subseteq \partial_{D} \Omega$, then a density lower bound à la De Giorgi-Carriero-Leaci for balls well contained in $\Omega$ would enforce the choice $\rho^{\prime \prime}=\rho_{k}^{\prime \prime}$ with $\rho_{k}^{\prime \prime} \rightarrow 0$ as $k \rightarrow+\infty$, and the resulting estimate would be useless.

Remark 5.9. Notice finally that for $A \subseteq \Omega$ open and connected with $\bar{A} \cap\left(\partial_{N} \Omega \cup\right.$ $\Gamma(t))=\emptyset$ but with $\mathcal{H}^{1}\left(\partial A \cap\left(\partial_{D} \Omega \backslash \Gamma(t)\right)\right)=0$, it is readily seen, as a consequence of global stability and since the boundary condition is not seen on $A$, that $u(t)$ is constant on $A$. In this case, following arguments similar to those in Step 4, one can show that for every $t \in[0, T]$ there exists $c_{A}^{k} \in \mathbb{R}$ such that $u^{k}(t)+c_{A}^{k} \rightarrow u(t)$ uniformly on $\bar{A}$.

We conclude the section by considering a strong formulation of the FrancfortMarigo model.

Definition 5.10 (Strong quasi-static evolutions). We say that a map $[0, T] \ni t \mapsto$ $(u(t), K(t))$ with

$$
K(t) \subseteq \bar{\Omega} \text { is closed and countably } \mathcal{H}^{1} \text {-rectifiable }
$$

and

$$
u(t) \in \mathcal{C}^{0}\left(\left(\Omega \cup \partial_{D} \Omega\right) \backslash K(t)\right) \cap \mathcal{C}^{1}(\Omega \backslash K(t)),
$$

is a quasi-static evolution in the strong sense relative to the boundary displacement $g$ if $u(t)=g(t)$ on $\partial_{D} \Omega \backslash K(t)$ for every $t \in[0, T]$, and the three following properties hold.

- Irreversibility: for every $0 \leq s \leq t \leq T$, then $K(s) \subseteq K(t)$.

- Global stability: for every $t \in[0, T]$, for every closed and countably $\mathcal{H}^{1}$ rectifiable set $K^{\prime} \subset \bar{\Omega}$ such that $K(t) \subseteq K^{\prime}$, and every function $v \in \mathcal{C}^{0}((\Omega \cup$ 
$\left.\left.\partial_{D} \Omega\right) \backslash K^{\prime}\right) \cap \mathcal{C}^{1}\left(\Omega \backslash K^{\prime}\right)$ satisfying $v=g(t)$ on $\partial_{D} \Omega \backslash K^{\prime}$, then

$$
\mathcal{E}(u(t), K(t)) \leq \mathcal{E}\left(v, K^{\prime}\right) .
$$

- Energy balance: for every $t \in[0, T]$

$$
\mathcal{E}(t)=\mathcal{E}(0)+\int_{0}^{t} \int_{\Omega \backslash K(s)} D W(\nabla u(x, s)) \cdot \nabla \dot{g}(s, x) d x d s .
$$

We speak about strong formulation because of the regularity of the cracks and of the fact that admissible displacements admit elastic strains in a classical sense (without resorting to weak notions of derivatives). Existence of quasi-static evolutions in a strong sense was established in [11] for $W(\xi)=|\xi|^{2}$ under the assumption that the number of connected components of the admissible closed cracks is a priori fixed.

The following result is a generalization of that in [11] without any assumption on the number of connected components of the cracks.

Theorem 5.11. Assume that (5.1), (5.2), (5.3) and (5.4) hold, and that $W \in \mathcal{C}^{1}\left(\mathbb{R}^{2}\right) \cap$ $\mathcal{C}^{2}\left(\mathbb{R}^{2} \backslash\{0\}\right)$ satisfies $\left(\mathrm{H}_{1}\right),\left(\mathrm{H}_{3}\right)$ and

$\left(\mathrm{H}_{4}\right)$ There exists $\lambda>0$ such that for every $\xi \in \mathbb{R}^{N} \backslash\{0\}$,

$$
\left|D^{2} W(\xi)\right| \leq \lambda|\xi|^{p-2}
$$

$\left(\mathrm{H}_{5}\right)$ There exists $\mu>0$ such that for every $\xi \in \mathbb{R}^{N} \backslash\{0\}$ and $\eta \in \mathbb{R}^{N}$,

$$
D^{2} W(\xi) \eta \cdot \eta \geq \mu|\xi|^{p-2}|\eta|^{2} .
$$

Then there exists a strong quasi-static evolution $[0, T] \ni t \mapsto(u(t), K(t))$ relative to the boundary displacement $g$ and such that $K(0)=\Gamma_{0} \cup \partial_{N} \Omega$.

Proof. Let $[0, T] \ni t \mapsto(u(t), \Gamma(t))$ be the regular quasi-static evolution given by Theorem 5.2. Thanks to the global stability of $(u(t), \Gamma(t))$, and in view of [13, Theorem 2], we have also (up to reducing $\alpha$ )

$$
u(t) \in \mathcal{C}_{\mathrm{loc}}^{1, \alpha}(\Omega \backslash \Gamma(t)) .
$$

To obtain a quasi-static evolution in the strong sense it suffices to set

$$
K(t):=\Gamma(t) \cup \partial_{N} \Omega .
$$

Indeed we only need to prove (5.22). Without loss of generality we can assume that $\mathcal{E}\left(v, K^{\prime}\right)<+\infty$ and $\|v\|_{\infty} \leq\|g(t)\|_{\infty}$. Then, since $\mathcal{H}^{1}\left(K^{\prime}\right)<+\infty$, we have $v \in S B V^{p}(\Omega)$ with $\mathcal{H}^{1}\left(S_{v} \backslash K^{\prime}\right)=0$ and $v=g(t)$ on $\partial_{D} \Omega \backslash K^{\prime}$. Hence (5.22) follows by the global stability (4.3) since $\left(v, K^{\prime}\right) \in \mathcal{A}(g(t))$ and $\Gamma(t) \widetilde{\subset} K^{\prime}$. 


\section{Density lower bound for the jump set of quasi-minimizers under homogeneous Dirichlet boundary conditions}

The object of this section is to prove Theorem 3.4 on which rests the analysis of Section 5. It states a density lower bound for the jump set of quasi-minimizers of suitable free discontinuity problems involved in the analysis of crack propagation. Throughout the section, we assume that $\Omega, \partial_{D} \Omega, \partial_{N} \Omega$ and $\Omega^{\prime}$ satisfy (3.1), $W$ satisfies $\left(\mathrm{H}_{1}\right)-\left(\mathrm{H}_{3}\right)$, while $\kappa$ satisfies (3.2).

Following the approach of $[6,12,15]$, the density lower bound is a consequence of a suitable decay estimate (see Lemma 6.6). The main difference with respect to the results of $[6,12]$ is that we allow balls to have center in $\Omega$ and to possibly intersect the Dirichlet part $\partial_{D} \Omega$ of the boundary: this fact is essential for our application to quasi-static crack propagation (see Remark 5.8). The proof of the decay estimate will rest on a regularity result for local minimizers of the bulk energy (see Theorem 3.8), whose proof will be given in Section 7. Note that our surface energy depends on the approximate normal to the crack, but it controls and is controlled by the surface measure $\mathcal{H}^{N-1}$ of the crack: as a consequence, this makes no essential difference with respect to the Mumford-Shah type functionals considered in the above mentioned papers.

We will follow closely [3, Sections 7.1 and 7.2], taking care of the necessary modifications required by the Dirichlet boundary condition. The decay estimate is proved by contradiction considering rescaled problems on the unit ball and studying in details their asymptotic properties. We collect such general properties in Subsection 6.1.

\subsection{Problems on the unit ball}

Given $D \subseteq B_{1}$ a Borel set, $c>0, u \in S B V^{p}\left(B_{1}\right)$ and $\rho \leq 1$, let us set

$$
F_{D}\left(u, c, B_{\rho}\right):= \begin{cases}\int_{B_{\rho}} W(\nabla u) d x+c \int_{S_{u} \cap B_{\rho}} \kappa\left(v_{u}\right) d \mathcal{H}^{N-1} & \text { if } u=0 \text { a.e. in } D \\ +\infty & \text { otherwise. }\end{cases}
$$

We denote by

$$
\boldsymbol{m}_{D}\left(u, c, B_{\rho}\right):=\inf \left\{F_{D}\left(v, c, B_{\rho}\right): v \in S B V^{p}\left(B_{1}\right),\{v \neq u\} \Subset B_{\rho}\right\}
$$

the minimal value, and by $\operatorname{Dev}_{D}\left(u, c, B_{\rho}\right)$ the deviation from minimality of $u$ on $B_{\rho}$ for $F_{D}$, defined, if $\boldsymbol{m}_{D}\left(u, c, B_{\rho}\right)<+\infty$, by

$$
\operatorname{Dev}_{D}\left(u, c, B_{\rho}\right):=F_{D}\left(u, c, B_{\rho}\right)-\boldsymbol{m}_{D}\left(u, c, B_{\rho}\right) .
$$

The following compactness result is a variant of [3, Proposition 7.5]. 
Lemma 6.1. Let $D_{h} \subseteq B_{1}$ be a sequence of Borel sets such that for some $d_{0}>0$ we have $\left|D_{h}\right| \geq d_{0}$ for every $h \in \mathbb{N}$. Let $\left(v_{h}\right) \subset S B V^{p}\left(B_{1}\right)$ be such that

$$
\sup _{h \in \mathbb{N}} \int_{B_{1}} W\left(\nabla v_{h}\right) d x<+\infty, \quad \mathcal{H}^{N-1}\left(S_{v_{h}}\right) \rightarrow 0, \quad v_{h}=0 \text { a.e. in } D_{h} .
$$

Let us define $\bar{v}_{h}:=\left(v_{h} \wedge \tau^{+}\left(v_{h}, B_{1}\right)\right) \vee \tau^{-}\left(v_{h}, B_{1}\right)$, where

$$
\left\{\begin{array}{l}
\tau^{-}\left(v_{h}, B_{1}\right):=\inf \left\{t \in[-\infty,+\infty]:\left|\left\{v_{h}<t\right\}\right| \geq\left[2 \gamma_{N} \mathcal{H}^{N-1}\left(S_{v_{h}}\right)\right]^{\frac{N}{N-1}}\right\}, \\
\tau^{+}\left(v_{h}, B_{1}\right):=\inf \left\{t \in[-\infty,+\infty]:\left|\left\{v_{h}<t\right\}\right| \geq\left|B_{1}\right|-\left[2 \gamma_{N} \mathcal{H}^{N-1}\left(S_{v_{h}}\right)\right]^{\frac{N}{N-1}}\right\},
\end{array}\right.
$$

and $\gamma_{N}>0$ is the dimensional constant of the isoperimetric inequality.

Then there exists $h_{0} \in \mathbb{N}$ (depending only on $d_{0}$ and $N$ ) such that $\bar{v}_{h}=0$ a.e. in $D_{h}$ for any $h \geq h_{0}$. Moreover there exist a subsequence $\left(v_{h_{j}}\right) \subset\left(v_{h}\right)$, and a function $v \in W^{1, p}\left(B_{1}\right)$ such that $\bar{v}_{h_{j}} \rightarrow v$ strongly in $L^{p}\left(B_{1}\right), v_{h_{j}} \rightarrow v$ a.e. in $B_{1}$, and

$$
\int_{B_{\rho}} W(\nabla v) d x \leq \liminf _{j \rightarrow+\infty} \int_{B_{\rho}} W\left(\nabla \bar{v}_{h_{j}}\right) d x \quad \text { for every } \rho \leq 1 .
$$

Proof. Let us show that the condition $\bar{v}_{h}=0$ a.e. in $D_{h}$ is satisfied for $h$ large enough. Since $v_{h}=0$ a.e. in $D_{h}$, this is a consequence of the fact that for $h$ large enough

$$
\tau^{-}\left(v_{h}, B_{1}\right) \leq 0 \quad \text { and } \quad \tau^{+}\left(v_{h}, B_{1}\right) \geq 0 .
$$

Indeed, given $\varepsilon>0$, since $\mathcal{H}^{N-1}\left(S_{v_{h}}\right) \rightarrow 0$ we have for $h \geq h_{0}$ independent of $\varepsilon$

$$
\left|\left\{v_{h}<\varepsilon\right\}\right| \geq\left|D_{h}\right| \geq d_{0}>\left[2 \gamma_{N} \mathcal{H}^{N-1}\left(S_{v_{h}}\right)\right]^{\frac{N}{N-1}} .
$$

The value $\varepsilon$ is thus admissible for the computation of $\tau^{-}\left(v_{h}, B_{1}\right)$ for $h \geq h_{0}$ so that $\tau^{-}\left(v_{h}, B_{1}\right) \leq \varepsilon$. Since $\varepsilon$ is arbitrary, we deduce that $\tau^{-}\left(v_{h}, B_{1}\right) \leq 0$. A similar proof shows that $\tau^{+}\left(v_{h}, B_{1}\right) \geq 0$, and thus (6.2) follows.

In view of [3, Proposition 7.5 and Remark 7.6], denoting by

$$
m_{h}:=\inf \left\{t \in[-\infty,+\infty]:\left|\left\{v_{h}>t\right\}\right| \leq\left|B_{1}\right| / 2\right\},
$$

a median for $v_{h}$, there exists a subsequence $\left(v_{h_{j}}\right)$ and a function $v \in W^{1, p}\left(B_{1}\right)$ such that

$$
\bar{v}_{h_{j}}-m_{h_{j}} \rightarrow v \quad \text { strongly in } L^{p}\left(B_{1}\right), \quad v_{h_{j}}-m_{h_{j}} \rightarrow v \quad \text { a.e. in } B_{1},
$$

and, applying Ambrosio's theorem to the truncation at level $M$ of the previous sequence, and letting $M \rightarrow+\infty$, we get for every $\rho \leq 1$

$$
\int_{B_{\rho}} W(\nabla v) d x \leq \liminf _{j \rightarrow+\infty} \int_{B_{\rho}} W\left(\nabla \bar{v}_{h_{j}}\right) d x .
$$


The proof of the lemma follows if we show that the sequence $\left(m_{h_{j}}\right)$ is bounded, since we can consider (up to extracting a further subsequence) $v+m$ as limit function, where $m$ is a limit point for $\left(m_{h_{j}}\right)$. Since $\bar{v}_{h}=0$ a.e. in $D_{h}$ for $h \geq h_{0}$, in view of (6.3), we obtain

$$
\limsup _{j \rightarrow+\infty}\left|m_{h_{j}}\right|^{p}\left|D_{h_{j}}\right| \leq\|v\|_{p}^{p}
$$

and the result follows since $\left|D_{h_{j}}\right| \geq d_{0}$.

The following proposition is an adaptation of [3, Theorem 7.7] to the case of a homogeneous Dirichlet boundary condition. It deals with the asymptotic behaviour of sequences of functions $v_{h}$ with vanishing deviation from minimality and vanishing jump set: as expected, the limit Sobolev function is a local minimizer of the integral functional associated to $W$ under a suitable homogeneous Dirichlet boundary condition.

Proposition 6.2. Let $f_{h}: \mathbb{R}^{N-1} \rightarrow \mathbb{R}$ be a sequence of continuous functions, and let $D_{h}:=\left\{x=\left(x^{\prime}, x_{N}\right) \in B_{1}: x_{N} \leq f_{h}\left(x^{\prime}\right)\right\}$. Assume that $\left(f_{h}\right)$ is locally uniformly converging to the constant function $-\delta$, with $\delta \in[0,1)$. Let $c_{h}>0$ and $v_{h} \in S B V^{p}\left(B_{1}\right)$ be such that

$$
\begin{gathered}
\sup _{h \in \mathbb{N}} F_{D_{h}}\left(v_{h}, c_{h}, B_{1}\right)<+\infty, \\
\lim _{h \rightarrow+\infty} \operatorname{Dev}_{D_{h}}\left(v_{h}, c_{h}, B_{1}\right)=0, \\
\lim _{h \rightarrow+\infty} \mathcal{H}^{N-1}\left(S_{v_{h}}\right)=0, \\
v_{h} \rightarrow v \in W^{1, p}\left(B_{1}\right) \quad \text { a.e. in } B_{1} .
\end{gathered}
$$

Then $v$ is a local minimizer of $F_{0, \delta}\left(\cdot, B_{1}\right)$ (see Definition 3.6), and

$$
\lim _{h \rightarrow+\infty} F_{D_{h}}\left(v_{h}, c_{h}, B_{\rho}\right)=\int_{B_{\rho}} W(\nabla v) d x \quad \text { for every } \rho \in(0,1) .
$$

Proof. Since $\rho \mapsto F_{D_{h}}\left(v_{h}, c_{h}, B_{\rho}\right)$ is increasing on $[0,1]$, by Helly's theorem we may assume that up to a subsequence

$$
\lim _{h \rightarrow+\infty} F_{D_{h}}\left(v_{h}, c_{h}, B_{\rho}\right)=\alpha(\rho) \quad \text { for every } \rho \in[0,1]
$$

for some increasing function $\alpha:[0,1] \rightarrow[0,+\infty)$.

Observe that since $f_{h} \rightarrow-\delta$ locally uniformly, and $\delta<1$, then necessarily $\left|D_{h}\right| \geq d_{0}$ for some constant $d_{0}>0$. Hence by Lemma 6.1 we have that (for a not relabeled subsequence) $\bar{v}_{h} \rightarrow v$ strongly in $L^{p}\left(B_{1}\right), \bar{v}_{h}=0$ a.e. in $D_{h}$ for $h$ large enough, and for all $\rho \leq 1$

$$
\int_{B_{\rho}} W(\nabla v) d x \leq \liminf _{h \rightarrow+\infty} \int_{B_{\rho}} W\left(\nabla \bar{v}_{h}\right) d x .
$$


We claim that

$$
\lim _{h \rightarrow+\infty} F_{D_{h}}\left(\bar{v}_{h}, c_{h}, B_{\rho}\right)=\alpha(\rho) \quad \text { for a.e. } \rho \in(0,1)
$$

and that

$$
\lim _{h \rightarrow+\infty} \operatorname{Dev}_{D_{h}}\left(\bar{v}_{h}, c_{h}, B_{\rho}\right)=0 \quad \text { for every } \rho \in(0,1) .
$$

Assuming the validity of (6.6) and (6.7), let us show that $v \in W^{1, p}\left(B_{1}\right)$ is a local minimizer of the functional $F_{0, \delta}\left(\cdot, B_{1}\right)$ given in (3.4). Let $w \in W^{1, p}\left(B_{1}\right)$ with $\{w \neq v\} \Subset B_{1}$ and $w=0$ a.e. in $B_{1} \backslash H_{\delta}$. In view of Lemma 6.3 below, we can find $w_{h} \in W^{1, p}\left(B_{1}\right)$ satisfying $w_{h}=0$ a.e. in $D_{h}$, and $w_{h} \rightarrow w$ strongly in $W^{1, p}\left(B_{1}\right)$.

Let $0<\rho^{\prime}<\rho<1$ be such that $\alpha$ is continuous at $\rho,\{w \neq v\} \Subset B_{\rho^{\prime}}$ and (6.6) holds for both $\rho$ and $\rho^{\prime}$. Then we have the inequality

$$
\begin{aligned}
F_{D_{h}}\left(\bar{v}_{h}, c_{h}, B_{\rho}\right) \leq & F_{0, \delta}\left(w_{h}, B_{\rho^{\prime}}\right)+\operatorname{Dev}_{D_{h}}\left(\bar{v}_{h}, c_{h}, B_{\rho}\right) \\
& +C\left[F_{D_{h}}\left(\bar{v}_{h}, c_{h}, B_{\rho} \backslash B_{\rho^{\prime}}\right)+F_{0, \delta}\left(w_{h}, B_{\rho} \backslash B_{\rho^{\prime}}\right)\right] \\
& +\frac{C}{\left(\rho-\rho^{\prime}\right)^{p}} \int_{B_{\rho} \backslash B_{\rho^{\prime}}}\left|\bar{v}_{h}-w_{h}\right|^{p} d x,
\end{aligned}
$$

where $C>0$ depends only on $p$ and $L$. This inequality is obtained by the same proof of [3, Lemma 7.4], i.e., by comparing $\bar{v}_{h}$ with $\eta w_{h}+(1-\eta) \bar{v}_{h}$, with $\eta$ a suitable cut-off between $B_{\rho^{\prime}}$ and $B_{\rho}$ : note that $\eta w_{h}+(1-\eta) \bar{v}_{h}=0$ a.e. in $D_{h}$ as both $w_{h}$ and $\bar{v}_{h}$ vanish on that set. For $h \rightarrow+\infty$, in view of our choice of $\rho$ and $\rho^{\prime}$, and thanks to (6.7) we obtain that

$$
\begin{aligned}
\alpha(\rho) \leq & \int_{B_{\rho^{\prime}}} W(\nabla w) d x+C\left[\alpha(\rho)-\alpha\left(\rho^{\prime}\right)+\int_{B_{\rho} \backslash B_{\rho^{\prime}}} W(\nabla w) d x\right] \\
& +\frac{C}{\left(\rho-\rho^{\prime}\right)^{p}} \int_{B_{\rho} \backslash B_{\rho^{\prime}}}|v-w|^{p} d x .
\end{aligned}
$$

Since $w=v$ on $B_{\rho} \backslash B_{\rho^{\prime}}$, letting $\rho^{\prime} \nearrow \rho$ we get

$$
\alpha(\rho) \leq \int_{B_{\rho}} W(\nabla w) d x
$$

Choosing $w=v$ in the previous relation, we obtain in view of (6.5) and (6.6) that

$$
\alpha(\rho)=\int_{B_{\rho}} W(\nabla v) d x .
$$

Hence $F_{0, \delta}\left(v, B_{1}\right) \leq F_{0, \delta}\left(w, B_{1}\right)$, and the local minimality follows. Moreover, by (6.8), the monotone functions $\alpha$ and $\rho \mapsto \int_{B_{\rho}} W(\nabla v) d x$ coincides a.e. on 
$(0,1)$, and since the latter is continuous, they actually coincide everywhere on $(0,1)$. Hence $(6.4)$ holds.

In order to complete the proof, we need to show claims (6.6) and (6.7). We will use the fact that $\beta^{-1} \leq \kappa(v) \leq \beta$ for $v \in \mathbb{R}^{N}$ belonging to the unit sphere.

Let $\tilde{v}_{h}$ and $\tilde{\bar{v}}_{h}$ be the Lebesgue representatives of $v_{h}$ and $\bar{v}_{h}$ respectively. By definition of $\bar{v}_{h}$ and $\tau^{ \pm}\left(v_{h}, B_{1}\right)$ (see (6.1)), we have (see [3, Remark 7.6])

$c_{h} \int_{0}^{1} \mathcal{H}^{N-1}\left(\left\{\tilde{v}_{h} \neq \tilde{\bar{v}}_{h}\right\} \cap \partial B_{\rho}\right) d \rho=c_{h}\left|\left\{\tilde{v}_{h} \neq \tilde{\bar{v}}_{h}\right\}\right| \leq 2 c_{h}\left[2 \gamma_{N} \mathcal{H}^{N-1}\left(S_{v_{h}}\right)\right]^{\frac{N}{N-1}} \rightarrow 0$

where we used the fact that $c_{h} \mathcal{H}^{N-1}\left(S_{v_{h}}\right)$ is uniformly bounded. It yields, up to a further subsequence, that

$$
\lim _{h \rightarrow+\infty} c_{h} \mathcal{H}^{N-1}\left(\left\{\tilde{v}_{h} \neq \tilde{\bar{v}}_{h}\right\} \cap \partial B_{\rho}\right)=0 \quad \text { for a.e. } \rho \in(0,1) .
$$

Since for every $h \in \mathbb{N}$ and for a.e. $\rho \in(0,1)$ we have that $\mathcal{H}^{N-1}\left(S_{\bar{v}_{h}} \cap \partial B_{\rho}\right)=0$, we can write for $\rho<\rho^{\prime}<1$

$$
\begin{aligned}
F_{D_{h}}\left(\bar{v}_{h}, c_{h}, B_{\rho}\right) \leq & F_{D_{h}}\left(v_{h}, c_{h}, B_{\rho}\right) \\
\leq & F_{D_{h}}\left(\bar{v}_{h}, c_{h}, B_{\rho}\right)+\beta c_{h} \mathcal{H}^{N-1}\left(\left\{\tilde{v}_{h} \neq \tilde{\bar{v}}_{h}\right\} \cap \partial B_{\rho}\right) \\
& +\operatorname{Dev}_{D_{h}}\left(v_{h}, c_{h}, B_{\rho^{\prime}}\right) .
\end{aligned}
$$

The first inequality comes by truncation. The second one follows by the same proof of [3, Lemma 7.3] which is based on a comparison between $v_{h}$ and $\bar{v}_{h} \chi_{B_{\rho}}+$ $v_{h} \chi_{B_{\rho^{\prime}} \backslash B_{\rho}}$ : note that the last function vanishes on $D_{h}$, so that it is an admissible competitor. Letting $h \rightarrow+\infty$ we obtain (6.6).

For what concerns (6.7), we observe that

$$
\begin{aligned}
\operatorname{Dev}_{D_{h}}\left(\bar{v}_{h}, c_{h}, B_{\rho}\right) \leq & F_{D_{h}}\left(\bar{v}_{h}, c_{h}, B_{\rho}\right)-F_{D_{h}}\left(v_{h}, c_{h}, B_{\rho}\right) \\
& +\beta c_{h} \mathcal{H}^{N-1}\left(\left\{\tilde{v}_{h} \neq \tilde{\bar{v}}_{h}\right\} \cap \partial B_{\rho}\right)+\operatorname{Dev}_{D_{h}}\left(v_{h}, c_{h}, B_{\rho^{\prime}}\right) .
\end{aligned}
$$

This inequality follows by the same proof [3, Lemma 7.3] by taking as competitor $w \chi_{B_{\rho}}+v_{h} \chi_{B_{\rho^{\prime}} \backslash B_{\rho}}$, where $w \in S B V^{p}\left(B_{\rho}\right)$ is such that $\left\{w \neq \bar{v}_{h}\right\} \Subset B_{\rho}$ and $w=0$ a.e. in $D_{h}$. Sending $h \rightarrow+\infty$, we deduce that (6.7) holds for a.e. $\rho \in(0,1)$. Since the deviation is an increasing function of $\rho$, we deduce that (6.7) actually holds for every $\rho \in(0,1)$.

In the previous proof, we used the following technical:

Lemma 6.3. Let $f_{h}: \mathbb{R}^{N-1} \rightarrow \mathbb{R}$ be a sequence of continuous functions, and let $D_{h}:=\left\{x=\left(x^{\prime}, x_{N}\right) \in B_{1}: x_{N} \leq f_{h}\left(x^{\prime}\right)\right\}$. Assume that $\left(f_{h}\right)$ is locally uniformly converging to the constant function $-\delta$, with $\delta \in[0,1)$. For every $w \in W^{1, p}\left(B_{1}\right)$ with $w=0$ a.e. in $\left\{x=\left(x^{\prime}, x_{N}\right) \in B_{1}: x_{N} \leq-\delta\right\}$, there exists $w_{h} \in W^{1, p}\left(B_{1}\right)$ with $w_{h}=0$ a.e. in $D_{h}$ and

$$
w_{h} \rightarrow w \quad \text { strongly in } W^{1, p}\left(B_{1}\right) .
$$


Proof. Let us consider

$$
U:=\left\{x=\left(x^{\prime}, x_{N}\right) \in B_{1}: x_{N} \geq-\delta\right\} \cup\left((-2,2)^{N-1} \times(-2,-\delta)\right) .
$$

We can extend $w$ to $U$ by setting $w=0$ outside $B_{1}$. Then, since $U$ enjoys the exterior cone condition, there exists $\hat{w} \in W^{1, p}\left(\mathbb{R}^{N}\right)$ such that $\hat{w}_{\mid U}=w$. We set for $x \in B_{1}$

$$
w_{h}(x):=\hat{w}\left(x-a_{h} e_{N}\right)
$$

where $a_{h}:=\sup _{\left|x^{\prime}\right| \leq 2}\left|f_{h}\left(x^{\prime}\right)+\delta\right| \rightarrow 0$. Clearly $w_{h} \in W^{1, p}\left(B_{1}\right), w_{h} \rightarrow w$ strongly in $W^{1, p}\left(B_{1}\right)$, and if $x=\left(x^{\prime}, x_{N}\right) \in D_{h}$, then

$$
x_{N}-a_{h} \leq x_{N}-f_{h}\left(x^{\prime}\right)+f_{h}\left(x^{\prime}\right)-a_{h} \leq-\delta
$$

so that $x-a_{h} e_{N} \in(-2,2)^{N-1} \times(-2,-\delta)$ and consequently $w_{h}(x)=0$.

\subsection{The density lower bound estimate}

In order to prove Lemma 6.6, we will need the following geometric fact:

Lemma 6.4. Let $\left(x_{h}\right)$ be a sequence in $\Omega \cup \partial_{D} \Omega$ such that $x_{h} \rightarrow \bar{x} \in \partial_{D} \Omega$, and let $\rho_{h} \rightarrow 0$ be such that $B_{\rho_{h}}\left(x_{h}\right) \subset \Omega^{\prime}$ and $B_{\rho_{h}}\left(x_{h}\right) \cap \partial_{D} \Omega \neq \emptyset$. Let us rescale $B_{\rho_{h}}\left(x_{h}\right)$ to the unit ball $B_{1}$ by means of the change of variable $x:=x_{h}+\rho_{h} y$ with $y \in B_{1}$, and let $D_{h} \subseteq B_{1}$ be the region corresponding to $\left(\Omega^{\prime} \backslash \bar{\Omega}\right) \cap B_{\rho_{h}}\left(x_{h}\right)$.

Then, there exists a coordinate system such that, up to a subsequence,

$$
D_{h}=\left\{y=\left(y^{\prime}, y_{N}\right) \in B_{1}: y_{N} \leq f_{h}\left(y^{\prime}\right)\right\}
$$

for some $f_{h} \in \mathcal{C}^{1}\left(\mathbb{R}^{N-1}\right)$ locally uniformly converging to a constant $-\delta$, with $\delta \in$ $[0,1]$.

Proof. Let us consider the orthogonal coordinate system relative to $\bar{x}$ such that

$$
\Omega \cap B_{r}(\bar{x})=\left\{x=\left(x^{\prime}, x_{N}\right) \in B_{r}(\bar{x}): x_{N}>f\left(x^{\prime}\right)\right\},
$$

where $r>0$ and $f \in \mathcal{C}^{1}\left(\mathbb{R}^{N-1}\right)$ is such that $f\left(\bar{x}^{\prime}\right)=\bar{x}_{N}$ and $\nabla f\left(\bar{x}^{\prime}\right)=0$. This is possible since $\partial_{D} \Omega$ is of class $\mathcal{C}^{1}$.

Since $x_{h} \rightarrow \bar{x}$ and $\rho_{h} \rightarrow 0$, then $B_{\rho_{h}}\left(x_{h}\right) \subseteq B_{r}(\bar{x})$ for $h$ large enough. Let us use the coordinate system in $\bar{x}$ also for defining the blow up: the region $D_{h}$ is then given for $h$ large by those $y=\left(y^{\prime}, y_{N}\right) \in B_{1}$ such that

$$
\left(x_{h}\right)_{N}+\rho_{h} y_{N} \leq f\left(x_{h}^{\prime}+\rho_{h} y^{\prime}\right),
$$

i.e.,

$$
y_{N} \leq f_{h}\left(y^{\prime}\right):=\frac{f\left(x_{h}^{\prime}+\rho_{h} y^{\prime}\right)-\left(x_{h}\right)_{N}}{\rho_{h}} .
$$


Let $z_{h}=\left(z_{h}^{\prime},\left(z_{h}\right)_{N}\right) \in B_{\rho_{h}}\left(x_{h}\right) \cap \partial_{D} \Omega$ so that we can write, as $\left(z_{h}\right)_{N}=f\left(z_{h}^{\prime}\right)$, and

$$
f_{h}\left(y^{\prime}\right)=\frac{f\left(x_{h}^{\prime}+\rho_{h} y^{\prime}\right)-f\left(x_{h}^{\prime}\right)+f\left(x_{h}^{\prime}\right)-f\left(z_{h}^{\prime}\right)+\left(z_{h}\right)_{N}-\left(x_{h}\right)_{N}}{\rho_{h}} .
$$

Since we have $\left|f\left(x_{h}^{\prime}\right)-f\left(z_{h}^{\prime}\right)\right| \leq C\left|x_{h}^{\prime}-z_{h}^{\prime}\right| \leq C \rho_{h}$ and $\left|\left(z_{h}\right)_{N}-\left(x_{h}\right)_{N}\right| \leq \rho_{h}$, then, up to a subsequence,

$$
\frac{f\left(x_{h}^{\prime}\right)-f\left(z_{h}^{\prime}\right)+\left(z_{h}\right)_{N}-\left(x_{h}\right)_{N}}{\rho_{h}} \rightarrow c \in \mathbb{R} .
$$

Moreover, the sequence of functions

$$
g_{h}\left(y^{\prime}\right):=\frac{f\left(x_{h}^{\prime}+\rho_{h} y^{\prime}\right)-f\left(x_{h}^{\prime}\right)}{\rho_{h}}
$$

converges locally uniformly to zero since for some $t_{h} \in(0,1)$

$$
\frac{f\left(x_{h}^{\prime}+\rho_{h} y^{\prime}\right)-f\left(x_{h}^{\prime}\right)}{\rho_{h}}=\nabla f\left(x_{h}^{\prime}+\rho_{h} t_{h} y^{\prime}\right) \cdot y^{\prime} \rightarrow \nabla f\left(\bar{x}^{\prime}\right) \cdot y^{\prime}=0 .
$$

We conclude that $f_{h} \rightarrow c$ locally uniformly on $\mathbb{R}^{N-1}$.

Since $f_{h}(0) \leq 0$, we infer that $c \leq 0$. On the other hand, since $D_{h} \neq \emptyset$, there exists $\xi_{h}=\left(\xi_{h}^{\prime},\left(\xi_{h}\right)_{N}\right) \in B_{1}$ with $f_{h}\left(\xi_{h}^{\prime}\right) \geq\left(\xi_{h}\right)_{N}>-1$, which easily entails that $c \geq-1$. The conclusion follows by taking $\delta=-c$.

Remark 6.5. From a geometrical point of view, the previous result shows that the rescaled version of $B_{\rho_{h}}\left(x_{h}\right) \cap \partial_{D} \Omega$ tends to a flat boundary. This property is standard if $x_{h} \equiv \bar{x} \in \partial_{D} \Omega$.

The following result is the key point to obtain a density lower bound for the jump set of quasi-minimizers of $F$.

Lemma 6.6 (Decay lemma). There exists a constant $C_{1}>0$ (depending only on $N, p, L, \mu)$ with the following property: for every $\tau \in(0,1)$ and $\eta>0$, there exist $\varepsilon(\tau, \eta)>0, \vartheta(\tau, \eta)>0$ and $r(\tau, \eta)>0$ such that for every ball $B_{\rho}(x) \subseteq$ $\Omega^{\prime}$ with $x \in \Omega \cup \partial_{D} \Omega, \rho \leq r(\tau, \eta)$, dist $\left(B_{\rho}(x), \partial_{N} \Omega\right)>\eta$, and for every $u \in$ $S B V^{p}\left(B_{\rho}(x)\right)$ satisfying $u=0$ a.e. in $\left(\Omega^{\prime} \backslash \bar{\Omega}\right) \cap B_{\rho}(x)$ with

$$
\mathcal{H}^{N-1}\left(S_{u} \cap B_{\rho}(x)\right) \leq \varepsilon(\tau, \eta) \rho^{N-1}, \quad \operatorname{Dev}\left(u, B_{\rho}(x)\right) \leq \vartheta(\tau, \eta) F\left(u, B_{\rho}(x)\right),
$$

then we have

$$
F\left(u, B_{\tau \rho}(x)\right) \leq C_{1} \tau^{N} F\left(u, B_{\rho}(x)\right)
$$


Proof. Let us proceed by contradiction by showing that the result holds true for any choice of

$$
C_{1}>\max \left\{4^{N}, C_{0}^{\prime} L\left(2^{N} L+\omega_{N}\right)\right\},
$$

where $L$ is the constant appearing in the growth estimate $\left(\mathrm{H}_{1}\right)$ for $W$, and $C_{0}^{\prime}$ is the constant given by Theorem 3.8 (with $R_{0}=3 / 4$, and for local minimizers whose $W^{1, p}\left(B_{1}\right)$ norm is less than or equal to $\left.L^{1 / p}\right)$.

Note that we can assume $\tau<1 / 4$ : indeed if $\tau \geq 1 / 4$, in view of the choice of $C_{1}$

$$
C_{1} \tau^{N} F\left(u, B_{\rho}(x)\right) \geq 4^{N} \frac{1}{4^{N}} F\left(u, B_{\rho}(x)\right) \geq F\left(u, B_{\tau \rho}(x)\right) .
$$

Assume by contradiction that there exist sequences of positive numbers $\varepsilon_{h}, \vartheta_{h}$, $r_{h} \rightarrow 0$, of points $x_{h} \in \Omega \cup \partial_{D} \Omega$, of radii $\rho_{h} \leq r_{h}$ such that $B_{\rho_{h}}\left(x_{h}\right) \subseteq \Omega^{\prime}$, $\operatorname{dist}\left(B_{\rho_{h}}\left(x_{h}\right), \partial_{N} \Omega\right)>\eta$, and of functions $u_{h} \in S B V^{p}\left(B_{\rho_{h}}\left(x_{h}\right)\right)$ with $u_{h}=0$ a.e. in $\left(\Omega^{\prime} \backslash \bar{\Omega}\right) \cap B_{\rho_{h}}\left(x_{h}\right)$, with the properties

$$
\begin{gathered}
\mathcal{H}^{N-1}\left(S_{u_{h}} \cap B_{\rho_{h}}\left(x_{h}\right)\right)=\varepsilon_{h} \rho_{h}^{N-1}, \\
\operatorname{Dev}\left(u_{h}, B_{\rho_{h}}\left(x_{h}\right)\right)=\vartheta_{h} F\left(u_{h}, B_{\rho_{h}}\left(x_{h}\right)\right),
\end{gathered}
$$

and

$$
F\left(u_{h}, B_{\tau \rho_{h}}\left(x_{h}\right)\right)>C_{1} \tau^{N} F\left(u_{h}, B_{\rho_{h}}\left(x_{h}\right)\right) .
$$

Following [3, Lemma 7.14] we rescale $B_{\rho_{h}}\left(x_{h}\right)$ to $B_{1}$ and $u_{h}$ to $v_{h} \in S B V^{p}\left(B_{1}\right)$ by setting

$$
v_{h}(y):=\rho_{h}^{(1-p) / p} c_{h}^{1 / p} u_{h}\left(x_{h}+\rho_{h} y\right)
$$

with

$$
c_{h}:=\frac{\rho_{h}^{N-1}}{F\left(u_{h}, B_{\rho_{h}}\left(x_{h}\right)\right)} .
$$

Let $D_{h} \subseteq B_{1}$ be the set associated to $\left(\Omega^{\prime} \backslash \bar{\Omega}\right) \cap B_{\rho_{h}}\left(x_{h}\right)$ under such a rescaling. It is not restrictive to assume that $D_{h} \neq \emptyset$. Indeed, if not, the Dirichlet boundary condition will not play a role, and the conclusion follows by [3, Lemma 7.14] (recall that the surface energy controls and is controlled by the surface area). Consequently, since $\rho_{h} \rightarrow 0$ and $\eta>0$, we can suppose, up to a subsequence, that $x_{h} \rightarrow \bar{x} \in$ $\partial_{D} \Omega$. Then by Lemma 6.4, up to a further subsequence, one can find a coordinate system in which

$$
D_{h}:=\left\{x=\left(x^{\prime} ; x_{N}\right) \in B_{1}: x_{N} \leq f_{h}\left(x^{\prime}\right)\right\},
$$

for some $f_{h} \in \mathcal{C}^{1}\left(\mathbb{R}^{N-1}\right)$ with $f_{h} \rightarrow-\delta$ locally uniformly, where $\delta \in[0,1]$.

With the notation of the beginning of Subsection 6.1, we get thanks to the $p$ homogeneity $\left(\mathrm{H}_{3}\right)$ of $W$ and since the surface energy depends only on the normal $v$,

$$
F_{D_{h}}\left(v_{h}, c_{h}, B_{1}\right)=1, \quad \operatorname{Dev}_{D_{h}}\left(v_{h}, c_{h}, B_{1}\right)=\vartheta_{h}, \quad \mathcal{H}^{N-1}\left(S_{v_{h}} \cap B_{1}\right)=\varepsilon_{h}
$$


and

$$
F_{D_{h}}\left(v_{h}, c_{h}, B_{\tau}\right)>C_{1} \tau^{N} .
$$

Let us assume first that $\delta \in(1 / 2,1]$. By [3, Proposition 7.5 and Remark 7.6], there exists $v \in W^{1, p}\left(B_{1 / 2}\right)$ such that, if $m_{h}$ is a median of $v_{h}$ in $B_{1 / 2}$, up to a subsequence

$$
v_{h}-m_{h} \rightarrow v \quad \text { a.e. in } B_{1 / 2} \text {. }
$$

There exists $h_{0} \in \mathbb{N}$ such that $D_{h} \cap B_{1 / 2}=\emptyset$ for all $h \geq h_{0}$. In particular, from (6.9) we obtain (we omit the subscript $D_{h}$ since the boundary condition disappears, and we localize on $B_{1 / 2}$ )

$$
F\left(v_{h}, c_{h}, B_{1 / 2}\right) \leq 1, \quad \operatorname{Dev}\left(v_{h}, c_{h}, B_{1 / 2}\right) \rightarrow 0, \quad \mathcal{H}^{N-1}\left(S_{v_{h}} \cap B_{1 / 2}\right) \rightarrow 0 .
$$

By [3, Theorem 7.7], $v$ is a local minimizer of $F_{0, \delta}\left(\cdot, B_{1 / 2}\right)$ and for all $\rho<1 / 2$

$$
\int_{B_{\rho}} W(\nabla v) d x=\lim _{h \rightarrow+\infty} F\left(v_{h}, c_{h}, B_{\rho}\right)=\lim _{h \rightarrow+\infty} F_{D_{h}}\left(v_{h}, c_{h}, B_{\rho}\right) \leq 1 .
$$

Next, according to Theorem 3.7 and in view of (3.5), the function $v$ turns out to be locally Lipschitz on $B_{1 / 2}$ with the estimate

$$
\underset{B_{1 / 4}}{\operatorname{ess} \sup }|\nabla v|^{p} \leq \frac{C_{0} 2^{N}}{\omega_{N}} \int_{B_{1 / 2}}|\nabla v|^{p} d x \leq \frac{C_{0}^{\prime} 2^{N} L}{\omega_{N}},
$$

where we used (6.11) and the growth condition $\left(\mathrm{H}_{1}\right)$ in the last inequality. But since $\tau<1 / 4$

$$
\lim _{h \rightarrow+\infty} F_{D_{h}}\left(v_{h}, c_{h}, B_{\tau}\right)=\int_{B_{\tau}} W(\nabla v) d x \leq L \int_{B_{\tau}}|\nabla v|^{p} d x \leq C_{0}^{\prime} 2^{N} L^{2} \tau^{N}
$$

which is against (6.10) since by construction $C_{1}>C_{0}^{\prime} 2^{N} L^{2}$.

If rather $0 \leq \delta \leq 1 / 2$, then $\left|D_{h}\right| \geq d_{0}>0$ for some $d_{0}>0$. By Lemma 6.1 and Proposition 6.2 there exists a local minimizer $v \in W^{1, p}\left(B_{1}\right)$ of $F_{0, \delta}\left(\cdot, B_{1}\right)$ such that, up to a subsequence, $v_{h} \rightarrow v$ a.e. in $B_{1}$. Moreover for every $\rho \in(0,1)$

$$
\int_{B_{\rho}} W(\nabla v) d x=\lim _{h \rightarrow+\infty} F_{D_{h}}\left(v_{h}, c_{h}, B_{\rho}\right) \leq 1 .
$$

Notice that $\|\nabla v\|_{p} \leq L^{1 / p}$, which is consistent with our choice of $C_{1}$. Then using the gradient bound given by Theorem 3.8, we have that

$$
\underset{B_{1 / 4}}{\operatorname{ess} \sup }|\nabla v|^{p} \leq C_{0}^{\prime}\left(\frac{2^{N}}{\omega_{N}} \int_{B_{1 / 2}}|\nabla v|^{p} d x+1\right) \leq C_{0}^{\prime}\left(\frac{2^{N} L}{\omega_{N}}+1\right),
$$

where we used (6.12) and the growth condition $\left(\mathrm{H}_{1}\right)$ to get the last inequality. Then we reach again a contradiction by arguing as in the previous case. 
We are now in a position to prove Theorem 3.4.

Proof of Theorem 3.4. In view of Lemma 6.6, we can adapt the proof of [3, Theorem 7.21] to our context: suitable modifications are needed to take into account the more general notion of quasi-minimizer, and the anisotropic surface energy.

Let us fix $\eta>0$. Let $A \subseteq \Omega^{\prime}$ be an open set, and let $u \in S B V^{p}(A)$ be a quasi-minimizer of $F(\cdot, A)$ with constants $s$ and $\omega$. Note that it is easily proved that

$$
F\left(u, B_{\rho}(x)\right) \leq \beta N \omega_{N} \rho^{N-1}+\omega \rho^{N-1+s}
$$

for any ball $B_{\rho}(x) \subseteq A$ with $\operatorname{dist}\left(B_{\rho}(x), \partial_{N} \Omega\right)>\eta$ by comparing $u$ with $u \chi_{B_{\rho}(x) \backslash B_{\rho^{\prime}}(x)}$ for $\rho^{\prime}<\rho$, and letting $\rho^{\prime} \nearrow \rho$.

Following the notation of Lemma 6.6, let $\tau \in(0,1)$ be such that $C_{1} \tau^{N} \leq$ $\tau^{N-1+s}$, and let $\sigma \in(0,1)$ be such that $C_{1} \sigma\left(\beta N \omega_{N}+1\right) \leq \beta^{-1} \varepsilon(\tau, \eta)$. Let us define

$\rho_{0}:=\min \left\{\frac{1}{\omega}, \frac{\beta^{-1} \varepsilon(\tau, \eta) \tau^{N} \vartheta(\tau, \eta)}{\omega}, \frac{\beta^{-1} \varepsilon(\tau, \eta) \sigma^{N-1} \vartheta(\sigma, \eta)}{\omega}, r(\sigma, \eta)^{s}, r(\tau, \eta)^{s}\right\}$,

and

$$
\vartheta_{0}:=\varepsilon(\sigma, \eta) .
$$

Note that in view of the decay lemma, the constants $\vartheta_{0}$ and $\rho_{0}$ only depend on $N$, $p, L, \mu, \beta, s, \omega$ and $\eta$.

The proof of the theorem proceeds by contradiction. Define the set

$$
I:=\left\{x \in A: \limsup _{\rho \rightarrow 0} \frac{1}{\omega_{N} \rho^{N}} \int_{B_{\rho}(x)}|u(y)|^{\frac{N}{N-1}} d y=+\infty\right\},
$$

and assume that we have

$$
\mathcal{H}^{N-1}\left(S_{u} \cap B_{\rho}(x)\right)<\vartheta_{0} \rho^{N-1}
$$

for some $x \in S_{u} \backslash I$, and some $\rho<\rho_{0}$ with $B_{\rho}(x) \subseteq A$ and $\operatorname{dist}\left(B_{\rho}(x), \partial_{N} \Omega\right)>\eta$.

We claim that

$$
F\left(u, B_{\sigma \tau^{h} \rho}(x)\right) \leq \beta^{-1} \varepsilon(\tau, \eta) \tau^{h s}\left(\sigma \tau^{h} \rho\right)^{N-1} \quad \text { for all } h \in \mathbb{N} .
$$

Let us prove $(6.15)$ by iteration.

Let $h=0$, and assume first that $\operatorname{Dev}\left(u, B_{\rho}(x)\right) \leq \vartheta(\sigma, \eta) F\left(u, B_{\rho}(x)\right)$. Since we have $\rho \leq r(\sigma, \eta)$ by our choice (6.14) of $\rho_{0}$, we deduce from Lemma 6.6 and the energy upper bound (6.13) that

$$
\begin{aligned}
F\left(u, B_{\sigma \rho}(x)\right) & \leq C_{1} \sigma^{N} F\left(u, B_{\rho}(x)\right) \leq C_{1} \sigma^{N}\left[\beta N \omega_{N} \rho^{N-1}+\omega \rho^{N-1+s}\right] \\
& \leq C_{1} \sigma(\sigma \rho)^{N-1}\left(\beta N \omega_{N}+1\right) \leq \beta^{-1} \varepsilon(\tau, \eta)(\sigma \rho)^{N-1} .
\end{aligned}
$$


If rather $\operatorname{Dev}\left(u, B_{\rho}(x)\right)>\vartheta(\sigma, \eta) F\left(u, B_{\rho}(x)\right)$, then using the definition of quasiminimizers

$$
\begin{aligned}
F\left(u, B_{\sigma \rho}(x)\right) & \leq F\left(u, B_{\rho}(x)\right) \leq \frac{1}{\vartheta(\sigma, \eta)} \operatorname{Dev}\left(u, B_{\rho}(x)\right) \\
& \leq \frac{\omega \rho^{N-1+s}}{\vartheta(\sigma, \eta)} \leq \beta^{-1} \varepsilon(\tau, \eta)(\sigma \rho)^{N-1},
\end{aligned}
$$

proving the validity of (6.15) for $h=0$.

Assume now that (6.15) holds for some $h \in \mathbb{N}$, and let us show that it still holds for $h+1$. Again, if $\operatorname{Dev}\left(u, B_{\sigma \tau^{h} \rho}(x)\right) \leq \vartheta(\tau, \eta) F\left(u, B_{\sigma \tau^{h} \rho}(x)\right)$, since $\sigma \tau^{h} \rho \leq$ $r(\tau, \eta)$, we can apply Lemma 6.6 to get that

$$
\begin{aligned}
F\left(u, B_{\sigma \tau^{h+1} \rho}(x)\right) & \leq C_{1} \tau^{N} F\left(u, B_{\sigma \tau^{h} \rho}(x)\right) \leq \beta^{-1} \varepsilon(\tau, \eta) C_{1} \tau^{N} \tau^{h s}\left(\sigma \tau^{h} \rho\right)^{N-1} \\
& \leq \beta^{-1} \varepsilon(\tau, \eta) \tau^{(h+1) s}\left(\sigma \tau^{h+1} \rho\right)^{N-1} .
\end{aligned}
$$

On the other hand, if $\operatorname{Dev}\left(u, B_{\sigma \tau^{h} \rho}(x)\right)>\vartheta(\tau, \eta) F\left(u, B_{\sigma \tau^{h} \rho}(x)\right)$, since $u$ is a quasi-minimizer, we get that

$$
\begin{aligned}
F\left(u, B_{\sigma \tau^{h+1} \rho}(x)\right) & \leq F\left(u, B_{\sigma \tau^{h} \rho}(x)\right) \leq \frac{1}{\vartheta(\tau, \eta)} \operatorname{Dev}\left(u, B_{\sigma \tau^{h} \rho}(x)\right) \\
& \leq \frac{\omega\left(\sigma \tau^{h} \rho\right)^{N-1+s}}{\vartheta(\tau, \eta)} \\
& \leq \beta^{-1} \varepsilon(\tau, \eta) \tau^{(h+1) s}\left(\sigma \tau^{h+1} \rho\right)^{N-1},
\end{aligned}
$$

which completes the proof of (6.15).

As a consequence of (6.15), we infer that for every $x \in S_{u} \backslash I$,

$$
\lim _{r \rightarrow 0} \frac{1}{r^{N-1}}\left(\int_{B_{r}(x)}|\nabla u|^{p} d y+\mathcal{H}^{N-1}\left(S_{u} \cap B_{r}(x)\right)\right)=0,
$$

which leads to a contradiction according to [3, Theorem 7.8]. Hence the conclusion of Theorem 3.4 holds for any $x \in S_{u} \backslash I$, and by density for any $x \in \overline{S_{u} \backslash I}$. Consequently, it suffices to show that $\overline{S_{u} \backslash I}=\overline{S_{u}}$. Consider $x \notin \overline{S_{u} \backslash I}$. By [3, Lemma 3.75] we have $\mathcal{H}^{N-1}(I)=0$, so that it is possible to find an open set $U \subseteq A$ containing $x$ for which $\mathcal{H}^{N-1}\left(S_{u} \cap U\right)=0$, and thus $u \in W^{1, p}(U)$. According to the Poincaré-Wirtinger inequality, the energy upper bound (6.13), and the coercivity condition $\left(\mathrm{H}_{1}\right)$, for any ball $B_{r}\left(x_{0}\right) \subseteq U$ we get that

$$
\int_{B_{r}\left(x_{0}\right)}\left|u(y)-\bar{u}_{x_{0}, r}\right|^{p} d y \leq c r^{p} \int_{B_{r}\left(x_{0}\right)}|\nabla u|^{p} d y \leq c^{\prime} r^{N-1+p},
$$

where $\bar{u}_{x_{0}, r}$ is the average of $u$ over $B_{r}\left(x_{0}\right)$. Thanks to the Campanato theorem (see e.g. [3, Theorem 7.51], we deduce that $u \in \mathcal{C}_{\text {loc }}^{0,1-1 / p}(U)$ which shows that $U \subseteq A \backslash S_{u}$. Hence $x \notin \overline{S_{u}}$ which completes the proof of Theorem 3.4. 


\section{Gradient bound estimate for local minimizers of integral functionals}

The object of this section is to prove the gradient bound estimate given by Theorem 3.8 for local minimizers of integral functionals on the intersection of the unit ball $B_{1}$ and a half plane of the type

$$
H_{\delta}:=\left\{x \in \mathbb{R}^{N}: x_{N}>-\delta\right\}
$$

where $\delta \in[0,1 / 2]$. This result - which was used in the proof of the decay lemma (Lemma 6.6) - is a generalization of [15, Theorem 2.2] involving a homogeneous Dirichlet boundary condition on a flat boundary.

The proof of Theorem 3.8 will be achieved through an approximation of the energy density $W$ by a sequence of non degenerate energies $W_{\varepsilon}$, and by considering associated integral functionals involving the local minimizer $u$ of the initial problem as boundary datum. We collect the properties of these auxiliary problems in Subsection 7.2, after having proved a suitable bound for the $L^{\infty}$ norm of $u$ in Subsection 7.1: the key point is that the associated estimates turn out to be independent of $\varepsilon$, so that the proof of Theorem 3.8 can be recovered by letting $\varepsilon \rightarrow 0$. It is also important for the application of the gradient bound to the proof of Theorem 3.4 (see in particular Lemma 6.6) that the estimates are independent of $\delta$.

\subsection{Some properties of the local minimizer}

Let $W: \mathbb{R}^{N} \rightarrow \mathbb{R}$ be a continuous function satisfying $\left(\mathrm{H}_{1}\right)$ and $\left(\mathrm{H}_{2}\right)$, and let $u \in W^{1, p}\left(B_{1}\right)$ with $u=0$ a.e. in $B_{1} \backslash H_{\delta}$ be such that $u$ is a local minimizer of $F_{0, \delta}$, i.e.,

$$
\int_{B_{1}} W(\nabla u) d x \leq \int_{B_{1}} W(\nabla z) d x
$$

for every $z \in W^{1, p}\left(B_{1}\right)$ with $\{z \neq u\} \Subset B_{1}$ and $z=0$ on $B_{1} \backslash H_{\delta}$.

We first establish a local boundedness property for the local minimizer.

Proposition 7.1. For every $1 / 2<R_{0}<1$, there exists a constant $c=c\left(N, p, L, R_{0}\right)>$ 0 such that

$$
\|u\|_{L^{\infty}\left(B_{R_{0}}\right)} \leq c\|\nabla u\|_{L^{p}\left(B_{1} ; \mathbb{R}^{N}\right)} .
$$

Proof. Let us first treat the case $p \leq N$. Given $B_{s}\left(x_{0}\right) \subset B_{t}\left(x_{0}\right) \subset B_{1}$ and $k \geq 0$, let us consider the function

$$
z:=u-\eta(u-k)_{+}
$$

where $\eta \in \mathcal{C}_{c}^{\infty}\left(B_{1}\right), 0 \leq \eta \leq 1, \eta=1$ on $B_{s}\left(x_{0}\right), \eta=0$ on $B_{1} \backslash B_{t}\left(x_{0}\right)$, $|\nabla \eta| \leq 2(t-s)^{-1}$. Notice that $z \in W^{1, p}\left(B_{1}\right)$ is such that $z=u$ on $B_{1} \backslash B_{t}\left(x_{0}\right)$, and that $z=0$ on $B_{1} \backslash H_{\delta}$ since $k \geq 0$. Thus $z$ is admissible for (7.1). By comparing $u$ and $z$ we obtain the following Caccioppoli inequality

$$
\int_{B_{s}\left(x_{0}\right) \cap\{u \geq k\}}|\nabla u|^{p} d x \leq \frac{c}{(t-s)^{p}} \int_{B_{t}\left(x_{0}\right) \cap\{u \geq k\}}(u-k)^{p} d x,
$$


where $c=c(N, p, L)>0$. Similarly (changing the definition of $z$ ), for every $k \leq 0$ we get

$$
\int_{B_{S}\left(x_{0}\right) \cap\{u \leq k\}}|\nabla u|^{p} d x \leq \frac{c}{(t-s)^{p}} \int_{B_{t}\left(x_{0}\right) \cap\{u \leq k\}}(k-u)^{p} d x .
$$

This means that $u$ belongs to a suitable De Giorgi class (see [18, Definition 7.1]): as a consequence the following inequality holds (see [18, Theorem 7.2])

$$
\|u\|_{L^{\infty}\left(B_{R_{0}}\right)} \leq\left(\frac{c}{\left(1-R_{0}\right)^{N}} \int_{B_{1}}|u|^{p} d x\right)^{1 / p},
$$

where $c=c(N, p, L)^{1}$. Since $\delta \leq 1 / 2$, then $u=0$ a.e. in $B_{1} \backslash H_{1 / 2}$. Thus according to the Poincare inequality, we infer that (7.2) holds with a constant depending on $N, p, L$ and $R_{0}$, but independent of $\delta$.

The case $p>N$ is immediate thanks the Sobolev embedding $W^{1, p}\left(B_{1}\right) \hookrightarrow$ $L^{\infty}\left(B_{1}\right)$ and the Poincaré inequality since $u=0$ a.e. on $B_{1} \backslash H_{1 / 2}$. In this last case, $u$ is actually globally bounded on $B_{1}$.

\subsection{Gradient bound for minimizers of a non-degenerate auxiliary problem}

Let us consider a non-degenerate density $f \in \mathcal{C}^{2}\left(\mathbb{R}^{N}\right)$ satisfying the following properties:

(A $\left.\mathrm{A}_{1}\right)$ There exist $\varepsilon \in(0,1), L>0$ and $p>1$ such that for every $\xi \in \mathbb{R}^{N}$,

$$
L^{-1}\left(\varepsilon^{2}+|\xi|^{2}\right)^{p / 2} \leq f(\xi) \leq L\left(\varepsilon^{2}+|\xi|^{2}\right)^{p / 2}
$$

(A2) There exists $\lambda>0$ such that for every $\xi \in \mathbb{R}^{N}$,

$$
\left|D^{2} f(\xi)\right| \leq \lambda\left(\varepsilon^{2}+|\xi|^{2}\right)^{(p-2) / 2} ;
$$

$\left(\mathrm{A}_{3}\right)$ There exists $\mu>0$ such that for every $\xi$ and $\eta \in \mathbb{R}^{N}$,

$$
D^{2} f(\xi) \eta \cdot \eta \geq \mu\left(\varepsilon^{2}+|\xi|^{2}\right)^{(p-2) / 2}|\eta|^{2} .
$$

In particular, since $f$ is convex from $\left(\mathrm{A}_{3}\right)$, we get from [18, Lemma 5.2] that

$$
|D f(\xi)| \leq c\left(\varepsilon^{2}+|\xi|^{2}\right)^{(p-1) / 2} \text { for all } \xi \in \mathbb{R}^{N},
$$

where $c>0$ is a constant depending only on $p$ and $L$. Moreover, from $\left(\mathrm{A}_{1}\right)$, it follows that $f$ satisfies the following $p$-growth and $p$-coercivity conditions:

$$
L^{-1}|\xi|^{p} \leq f(\xi) \leq L\left(1+|\xi|^{p}\right) \quad \text { for all } \xi \in \mathbb{R}^{N}
$$

${ }^{1}$ The proof in [18] has actually been performed in the case $p<N$. However, the critical case $p=$ $N$ proceeds exactly in the same way by using the Sobolev imbedding $W^{1, N}\left(\mathbb{R}^{N}\right) \hookrightarrow L^{q}\left(\mathbb{R}^{N}\right)$ for any $p \leq q<+\infty$, instead of that $W^{1, p}\left(\mathbb{R}^{N}\right) \hookrightarrow L^{p^{*}}\left(\mathbb{R}^{N}\right)$ when $p<N$. 
Let us fix a radius $1 / 2<R_{0}<1$, and consider the solution $v \in W^{1, p}\left(B_{1}\right)$ of the following minimization problem:

$$
\min \left\{\int_{B_{1}} f(\nabla z) d x: z \in W^{1, p}\left(B_{1}\right), z=u \text { a.e. in } B_{1} \backslash\left(B_{R_{0}} \cap H_{\delta}\right)\right\} .
$$

Note that $v$ is a solution of the following Euler-Lagrange equation:

$$
\begin{cases}\operatorname{div}(D f(\nabla v))=0 & \text { in } \mathcal{D}^{\prime}\left(B_{R_{0}} \cap H_{\delta}\right), \\ v=u & \mathcal{H}^{N-1} \text {-a.e. on } \partial\left(B_{R_{0}} \cap H_{\delta}\right) .\end{cases}
$$

Proposition 7.2. There exists a constant $K_{0}=K_{0}\left(N, p, L, R_{0},\|\nabla u\|_{L^{p}\left(B_{1} ; \mathbb{R}^{N}\right)}\right)>$ 0 such that

$$
\|v\|_{L^{\infty}\left(B_{R_{0}}\right)} \leq K_{0}
$$

Proof. Let us first observe that from Proposition 7.1, there exists a constant $k_{0}$ (depending only on $N, p, L, R_{0}$ and $\|\nabla u\|_{L^{p}\left(B_{1} ; \mathbb{R}^{N}\right)}$ ) such that

$$
\|u\|_{L^{\infty}\left(B_{\left(R_{0}+1\right) / 2}\right)} \leq k_{0} .
$$

Let $p \leq N$. Given $B_{S}\left(x_{0}\right) \subset B_{t}\left(x_{0}\right) \subset B_{R_{0}}$ and $k \geq k_{0}$, let us consider the function

$$
z:=v-\eta(v-k)_{+},
$$

with $\eta \in \mathcal{C}_{c}^{\infty}\left(B_{1}\right), 0 \leq \eta \leq 1, \eta=1$ on $B_{s}\left(x_{0}\right), \eta=0$ on $B_{1} \backslash B_{t}\left(x_{0}\right)$, and $|\nabla \eta| \leq$ $2(t-s)^{-1}$. Notice that $z \in W^{1, p}\left(B_{1}\right)$ and that $z=u$ a.e. on $B_{1} \backslash\left(B_{R_{0}} \cap H_{\delta}\right)$. Indeed $v=u$ on $B_{1} \backslash\left(B_{R_{0}} \cap H_{\delta}\right)$, and consequently $(v-k)_{+}=0$ on $B_{t}\left(x_{0}\right) \backslash\left(B_{R_{0}} \cap H_{\delta}\right)$ since $k \geq k_{0}$ and $k_{0}$ satisfies (7.8). Thus $z$ is admissible for the minimum problem (7.5). By comparing $v$ and $z$ we obtain the following Caccioppoli inequality

$$
\int_{B_{S}\left(x_{0}\right) \cap\{v \geq k\}}|\nabla v|^{p} d x \leq \frac{c}{(t-s)^{p}} \int_{B_{t}\left(x_{0}\right) \cap\{v \geq k\}}(v-k)^{p} d x+c\left|B_{t} \cap\{v \geq k\}\right|,
$$

where $c=c(N, p, L)>0$. Similarly, for every $k \leq-k_{0}$ we get

$$
\int_{B_{s}\left(x_{0}\right) \cap\{v \leq k\}}|\nabla v|^{p} d x \leq \frac{c}{(t-s)^{p}} \int_{B_{t}\left(x_{0}\right) \cap\{v \leq k\}}(k-v)^{p} d x+c\left|B_{t} \cap\{v \leq k\}\right| .
$$

The function $v$ thus belongs to a suitable De Giorgi class (see [18, Definition 7.1]), so that following [18, Theorem 7.2], $v$ is bounded in $B_{R_{0}}$ with

$$
\|v\|_{L^{\infty}\left(B_{R_{0}}\right)} \leq c\left[\left(\frac{1}{\left(1-R_{0}\right)^{N}} \int_{B_{1}}|v|^{p} d x\right)^{1 / p}+k_{0}+1\right],
$$

where the constant $c$ depends only on $N, p$ and $L$. Since $\delta \leq 1 / 2$, then in particular $v=0$ a.e. in $B_{1} \backslash H_{1 / 2}$, so that according to Poincaré inequality

$$
\|v\|_{L^{\infty}\left(B_{R_{0}}\right)} \leq c_{1}\left(\|\nabla v\|_{L^{p}\left(B_{1} ; \mathbb{R}^{N}\right)}+k_{0}+1\right) \leq c_{2}\left(\|\nabla u\|_{L^{p}\left(B_{1} ; \mathbb{R}^{N}\right)}+k_{0}+1\right),
$$


the last inequality coming from the minimality of $v$ together with the $p$-growth and $p$-coercivity conditions (7.4) satisfied by $f$. Notice that $c_{2}>0$ depends only $N, p$, $L, R_{0}$ and $\|\nabla u\|_{L^{p}\left(B_{1} ; \mathbb{R}^{N}\right)}$, but is independent of $\delta$.

Finally, the case $p>N$ follows from the Sobolev embedding $W^{1, p}\left(B_{R_{0}}\right) \hookrightarrow$ $L^{\infty}\left(B_{R_{0}}\right)$ together with the Poincare inequality and the minimality of $v$.

In view of equation (7.6), using the difference quotient method (see e.g. [18, Sections 8.2 and 8.4]), we infer that for every $r<R_{0}$,

$$
v \in W^{2,2 \wedge p}\left(B_{r} \cap H_{\delta}\right),
$$

and according to [18, Section 8.5], we also have that

$$
v \in \mathcal{C}^{1, \alpha}\left(B_{r} \cap \overline{H_{\delta}}\right) \quad \text { for some } \alpha \in(0,1) .
$$

Arguing as in [18, Section 8.4] one can prove that $\nabla v$ is actually bounded on $B_{R_{0}} \cap \partial H_{\delta}$ by some constant $M_{0}>0$ depending only on $N, \mu$ and $\|v\|_{L^{\infty}\left(B_{R_{0}}\right)}$, but independent of $\delta$. Consequently, from (7.7), we deduce the following result.

Proposition 7.3. There exists $M_{0}=M_{0}\left(N, p, L, \mu, R_{0},\|\nabla u\|_{L^{p}\left(B_{1} ; \mathbb{R}^{N}\right)}\right)>0$ such that

$$
\sup _{B_{R_{0}} \cap \partial H_{\delta}}|\nabla v| \leq M_{0} .
$$

We are now in position to prove the following gradient bound estimate for $v$. The proof is inspired by [15, Theorem 2.2]: rather than a Moser iteration technique, we employ the De Giorgi scheme which we found easier to adapt to the case with homogeneous Dirichlet conditions.

Proposition 7.4. Given $1 / 2<R_{0}<1$, let $v \in W^{1, p}\left(B_{1}\right)$ be the minimizer of (7.5). There exists a constant $c\left(N, p, L, \mu, R_{0},\|\nabla u\|_{L^{p}\left(B_{1} ; \mathbb{R}^{N}\right)}\right)>0$ such that for every $r<\rho \leq R_{0}$

$$
\sup _{B_{r}}|\nabla v|^{p} \leq c\left(\frac{1}{(\rho-r)^{N}} \int_{B_{\rho}}\left(|\nabla v|^{p}+1\right) d x+1\right) .
$$

Proof. By the Euler-Lagrange equation (7.6), we have that

$$
\int_{B_{R_{0}}} D f(\nabla v) \cdot \nabla \varphi d x=0
$$

for all $\varphi \in \mathcal{C}_{c}^{1}\left(B_{R_{0}}\right)$ satisfying $\varphi=0$ in $B_{R_{0}} \backslash H_{\delta}$. Take as particular test function $\varphi:=\eta^{2} D_{s} \psi$, where $\eta \in \mathcal{C}_{c}^{1}\left(B_{R_{0}} ;[0,1]\right)$ is a cut-off function, $\psi \in \mathcal{C}^{2}\left(B_{R_{0}}\right)$ with 
$\psi=0$ in $B_{R_{0}} \backslash H_{\delta}$, and $s=1, \ldots, N$. Thanks to an integration by parts, we infer that

$$
\begin{aligned}
\int_{B_{R_{0}}} D_{i j}^{2} f(\nabla v) D_{j}\left(D_{s} v\right)\left(D_{i} \psi\right) \eta^{2} d x= & 2 \int_{B_{R_{0}}} \eta D_{i} f(\nabla v) D_{s} \psi D_{i} \eta d x \\
& -2 \int_{B_{R_{0}}} \eta D_{i} f(\nabla v) D_{i} \psi D_{s} \eta d x
\end{aligned}
$$

where, here and in the sequel, we use the summation convention on repeated indexes. Observe that, by density, we can consider test functions $\psi \in W_{\mathrm{loc}}^{1,2 \wedge p}\left(B_{R_{0}}\right)$ satisfying $\psi=0$ a.e. in $B_{R_{0}} \backslash H_{\delta}$. Let us define

$$
V:=\left(\varepsilon^{2}+|\nabla v|^{2}\right)^{p / 2}
$$

and fix $r<R_{0}$. Since $V$ is the composition of $\nabla v \in W^{1,2 \wedge p}\left(B_{r} \cap H_{\delta}\right)$ with the smooth function $t \mapsto\left(\varepsilon^{2}+t^{2}\right)^{p / 2}$, then $V \in W^{1,2 \wedge p}\left(B_{r} \cap H_{\delta}\right)$ as well. Next, we set for each $s=1, \ldots, N$ and $k \geq\left(\varepsilon^{2}+M_{0}^{2}\right)^{p / 2}=: \kappa_{0}$,

$$
\psi:= \begin{cases}(V-k)_{+} D_{s} v & \text { in } B_{R_{0}} \cap H_{\delta}, \\ 0 & \text { in } B_{R_{0}} \backslash H_{\delta} .\end{cases}
$$

Clearly $\psi \in W^{1,2 \wedge p}\left(B_{r} \cap H_{\delta}\right)$. Then according to (7.9) and the fact that $k \geq \kappa_{0}$, we obtain that the upper trace of $\psi$ on $B_{R_{0}} \cap \partial H_{\delta}$ vanishes, from which we deduce that $\psi \in W_{\mathrm{loc}}^{1,2 \wedge p}\left(B_{R_{0}}\right)$. As a consequence we are allowed to take it as test function in (7.10). Using the $(p-1)$-growth property (7.3) of $D f$, we obtain that

$$
\begin{aligned}
& \int_{B_{R_{0}}} D_{i j}^{2} f(\nabla v) D_{j}\left(D_{s} v\right) D_{i}\left(D_{s} v\right)(V-k)_{+} \eta^{2} d x \\
+ & \int_{B_{R_{0}} \cap\{V>k\}} D_{i j}^{2} f(\nabla v) D_{j}\left(D_{s} v\right)\left(D_{i} V\right)\left(D_{s} v\right) \eta^{2} d x \\
\leq & c(N, p, L) \int_{B_{R_{0}} \cap\{V>k\}} V^{\frac{p-1}{p}} \eta|\nabla \eta|\left[(V-k)_{+}\left|D^{2} v\right|+|\nabla V||\nabla v|\right] d x .
\end{aligned}
$$

Since $\left(D_{j}\left(D_{s} v\right)\right)\left(D_{s} v\right)=\frac{1}{2} D_{j}\left(|\nabla v|^{2}\right)=\frac{1}{2} D_{j}\left(V^{2 / p}\right)=\frac{1}{p} V^{(2-p) / p} D_{j} V$ and $|\nabla v| \leq V^{1 / p}$, using the lower bound $\left(\mathrm{A}_{3}\right)$ on $D^{2} f$ yields

$$
\begin{aligned}
& \mu \int_{B_{R_{0}}} V^{\frac{p-2}{p}}(V-k)_{+}\left|D^{2} v\right|^{2} \eta^{2} d x+\frac{\mu}{p} \int_{B_{R_{0}} \cap\{V>k\}}|\nabla V|^{2} \eta^{2} d x \\
\leq & c(N, p, L) \int_{B_{R_{0}} \cap\{V>k\}} V^{\frac{p-1}{p}} \eta|\nabla \eta|(V-k)_{+}\left|D^{2} v\right| d x \\
+ & c(N, p, L) \int_{B_{R_{0}} \cap\{V>k\}} V \eta|\nabla \eta||\nabla V| d x .
\end{aligned}
$$


Applying Young's inequality to both terms in the right hand side of the previous relation leads to

$$
\begin{aligned}
\frac{\mu}{2} \int_{B_{R_{0}}} V^{\frac{p-2}{p}}(V-k)_{+}\left|D^{2} v\right|^{2} \eta^{2} d x+\frac{\mu}{2 p} \int_{B_{R_{0}} \cap\{V>k\}}|\nabla V|^{2} \eta^{2} d x \\
\left.\leq \frac{c(N, p, L)}{\mu} \int_{B_{R_{0}} \cap\{V>k\}}|\nabla \eta|^{2}\left[(V-k)_{+} V\right)+V^{2}\right] d x,
\end{aligned}
$$

from which we deduce that

$$
\int_{B_{R_{0}} \cap\{V>k\}}|\nabla V|^{2} \eta^{2} d x \leq c(N, p, L, \mu) \int_{B_{R_{0}} \cap\{V>k\}}|\nabla \eta|^{2} V^{2} d x
$$

for every $k \geq \kappa_{0}$.

Let us consider $B_{s}\left(x_{0}\right) \subset B_{t}\left(x_{0}\right) \subset B_{R_{0}}$, and $\eta \in \mathcal{C}_{c}^{1}\left(B_{R_{0}}\right)$ such that $0 \leq \eta \leq$ $1, \eta=1$ on $B_{s}\left(x_{0}\right), \eta=0$ on $B_{R_{0}} \backslash B_{t}\left(x_{0}\right)$ and $|\nabla \eta| \leq 2(t-s)^{-1}$. Then we get from (7.11) that for every $k \geq \kappa_{0}$,

$$
\begin{aligned}
& \int_{B_{s}\left(x_{0}\right) \cap\{V>k\}}|\nabla V|^{2} d x \leq \frac{c(N, p, L, \mu)}{(t-s)^{2}} \int_{B_{t}\left(x_{0}\right) \cap\{V>k\}} V^{2} d x \\
\leq & c(N, p, L, \mu)\left(\frac{1}{(t-s)^{2}} \int_{B_{t}\left(x_{0}\right) \cap\{V>k\}}(V-k)^{2} d x+k^{2}(t-s)^{-2}\left|B_{t}\left(x_{0}\right) \cap\{V>k\}\right|\right) .
\end{aligned}
$$

We conclude that, up to the term $k^{2}(t-s)^{-2}\left|B_{t}\left(x_{0}\right) \cap\{V>k\}\right|$, the function $V$ satisfies an inequality similar to that defining De Giorgi classes. The form of this term can nevertheless be handled in the De Giorgi iteration scheme, so that following [18, Theorems 7.2, 7.3 and Corollary 7.1] we obtain for every $r<\rho \leq$ $R_{0}$

$$
\sup _{B_{r}} V \leq c(N, p, L, \mu)\left(\frac{1}{(\rho-r)^{N}} \int_{B_{\rho}} V d x+\left(\varepsilon^{2}+M_{0}^{2}\right)^{\frac{p}{2}}\right) .
$$

The proof of the proposition is now complete since $|\nabla v|^{p} \leq V \leq\left(1+|\nabla v|^{2}\right)^{p / 2}$, $\varepsilon \leq 1$, and $M_{0}$ is estimated in Proposition 7.3.

\subsection{Proof of Theorem 3.8}

We now complete the proof of Theorem 3.8, namely extending Proposition 7.4 to the local minimizer of integral functionals where the density satisfies the degenerate assumptions $\left(\mathrm{H}_{1}\right)$ and $\left(H_{2}\right)$. This will be done as in the proof of [15, Theorem 2.2] thanks to a suitable regularization of $W$. 
Indeed according to [15, Lemma 2.4], there exist a sequence $\left(W_{\varepsilon}\right) \subset \mathcal{C}^{2}\left(\mathbb{R}^{N}\right)$ and a constant $c=c(N, p)>0$ such that for every $\xi$ and $\eta \in \mathbb{R}^{N}$,

(i) $c^{-1} L^{-1}\left(\varepsilon^{2}+|\xi|^{2}\right)^{p / 2} \leq W_{\varepsilon}(\xi) \leq c L\left(\varepsilon^{2}+|\xi|^{2}\right)^{p / 2}$;

(ii) $\left|D^{2} W_{\varepsilon}(\xi)\right| \leq \lambda_{\varepsilon}\left(\varepsilon^{2}+|\xi|^{2}\right)^{(p-2) / 2}$, for some $\lambda_{\varepsilon}>0$;

(iii) $D^{2} W_{\varepsilon}(\xi) \eta \cdot \eta \geq c^{-1} \mu\left(\varepsilon^{2}+|\xi|^{2}\right)^{(p-2) / 2}|\eta|^{2}$;

(iv) $W_{\varepsilon} \rightarrow W$ locally uniformly on $\mathbb{R}^{N}$.

It suffices to prove the result for $1 / 2<R_{0}<1$. Let us denote by $v_{\varepsilon} \in W^{1, p}\left(B_{R_{0}} \cap\right.$ $H_{\delta}$ ) the unique solution of the minimization problem $\left(W_{\varepsilon}\right.$ is strictly convex $)$

$\min \left\{\int_{B_{R_{0}} \cap H_{\delta}} W_{\varepsilon}(\nabla z) d x: z \in W^{1, p}\left(B_{R_{0}} \cap H_{\delta}\right), z=u \mathcal{H}^{N-1}\right.$-a.e. on $\left.\partial\left(B_{R_{0}} \cap H_{\delta}\right)\right\}$.

Extending $v_{\varepsilon}$ to $B_{1}$ by setting $v_{\varepsilon}=u$ on $B_{1} \backslash\left(B_{R_{0}} \cap H_{\delta}\right)$, then $v_{\varepsilon}$ is actually a minimizer of

$$
\min \left\{\int_{B_{1}} W_{\varepsilon}(\nabla z) d x: z \in W^{1, p}\left(B_{1}\right), z=u \text { a.e. in } B_{1} \backslash\left(B_{R_{0}} \cap H_{\delta}\right)\right\} .
$$

According to Proposition 7.4, one can find a constant $c=c\left(N, p, L, \mu, R_{0}\right.$, $\left.\|\nabla u\|_{L^{p}\left(B_{1} ; \mathbb{R}^{N}\right)}\right)>0$ such that for every $r<\rho \leq R_{0}$

$$
\sup _{B_{r}}\left|\nabla v_{\varepsilon}\right|^{p} \leq c\left(\frac{1}{(\rho-r)^{N}} \int_{B_{\rho}}\left(\left|\nabla v_{\varepsilon}\right|^{p}+1\right) d x+1\right) .
$$

Hence the sequence $\left(v_{\varepsilon}\right)$ is bounded in $W^{1, p}\left(B_{1}\right)$ (compare with $u$ and use Poincare inequality) and in $W^{1, \infty}\left(B_{r}\right)$ (recall that $u$ is bounded on $B_{R_{0}}$ thanks to Proposition 7.1, and $v_{\varepsilon}$ coincides with $u$ on $\left.\partial\left(B_{R_{0}} \cap H_{\delta}\right)\right)$. Thus it is possible to find a subsequence (not relabeled) such that for every $r<R_{0}$

$$
v_{\varepsilon} \rightarrow v_{\infty} \quad \text { weakly* in } W^{1, \infty}\left(B_{r}\right),
$$

for some $v_{\infty} \in W^{1, p}\left(B_{1}\right) \cap W_{\text {loc }}^{1, \infty}\left(B_{R_{0}}\right)$. Clearly $v_{\infty}=u$ a.e. in $B_{1} \backslash\left(B_{R_{0}} \cap H_{\delta}\right)$.

Since $W_{\varepsilon} \rightarrow W$ uniformly on compact sets, and thanks to the minimality of $v_{\varepsilon}$, we infer that

$$
\begin{aligned}
\int_{B_{r} \cap H_{\delta}} W\left(\nabla v_{\infty}\right) d x \leq & \liminf _{\varepsilon \rightarrow 0} \int_{B_{r} \cap H_{\delta}} W\left(\nabla v_{\varepsilon}\right) d x=\liminf _{\varepsilon \rightarrow 0} \int_{B_{r} \cap H_{\delta}} W_{\varepsilon}\left(\nabla v_{\varepsilon}\right) d x \\
& \leq \liminf _{\varepsilon \rightarrow 0} \int_{B_{R_{0}} \cap H_{\delta}} W_{\varepsilon}(\nabla u) d x=\int_{B_{R_{0}} \cap H_{\delta}} W(\nabla u) d x .
\end{aligned}
$$

Letting $r \nearrow R_{0}$ in the left hand side of the previous inequality, and since $v_{\infty}=u$ a.e. in $B_{1} \backslash\left(B_{R_{0}} \cap H_{\delta}\right)$, we obtain

$$
\int_{B_{1}} W\left(\nabla v_{\infty}\right) d x \leq \int_{B_{1}} W(\nabla u) d x
$$


By the minimality of $u$ (see (7.1)) we infer that the previous inequality is indeed an equality. Since $W$ is strictly convex (using $\left(\mathrm{H}_{2}\right)$ and Remark 3.1), we deduce that $u=v_{\infty}$. Then according to (7.12) with $r=\rho / 2$ we get for every $\rho \leq R_{0}$ (up to modifying $c$, still depending only on $N, p, L, \mu, R_{0}$ and $\left.\|\nabla u\|_{L^{p}\left(B_{1} ; \mathbb{R}^{N}\right)}\right)$

$$
\begin{aligned}
\underset{B_{\rho / 2}}{\operatorname{ess} \sup |\nabla u|^{p} \leq \liminf _{\varepsilon \rightarrow 0} \sup _{B_{\rho / 2}}\left|\nabla v_{\varepsilon}\right|^{p}} & \leq \liminf _{\varepsilon \rightarrow 0} c\left(\frac{1}{\rho^{N}} \int_{B_{\rho}}\left|\nabla v_{\varepsilon}\right|^{p} d x+1\right) \\
& \leq \liminf _{\varepsilon \rightarrow 0} c\left(\frac{1}{\rho^{N}} \int_{B_{\rho}} W_{\varepsilon}\left(\nabla v_{\varepsilon}\right) d x+1\right) .
\end{aligned}
$$

Finally, using again the minimality property of $v_{\varepsilon}$ we obtain (up to modifying $c$ in an admissible way)

$$
\begin{aligned}
\underset{B_{\rho / 2}}{\operatorname{esssup}}|\nabla u|^{p} & \leq \liminf _{\varepsilon \rightarrow 0} c\left(\frac{1}{\rho^{N}} \int_{B_{\rho}} W_{\varepsilon}(\nabla u) d x+1\right) \\
& =c\left(\frac{1}{\rho^{N}} \int_{B_{\rho}} W(\nabla u) d x+1\right) \leq c\left(\frac{1}{\rho^{N}} \int_{B_{\rho}}|\nabla u|^{p} d x+1\right)
\end{aligned}
$$

and the proof of Theorem 3.8 is complete.

\section{References}

[1] L. Ambrosio and A. BRAIDES, Energies in SBV and variational models in fracture mechanics, In: "Homogenization and Applications to Material Sciences (Nice, 1995)", GAKUTO Internat. Ser. Math. Sci. Appl., Vol. 9, Gakkōtosho, Tokyo, 1995, 1-22.

[2] L. Ambrosio and D. Pallara, Partial regularity of free discontinuity problems I, Ann. Scuola Norm. Sup. Pisa Cl. Sci. (4) 24 (1997), 1-38.

[3] L. Ambrosio, N. Fusco and D. Pallara, "Functions of Bounded Variation and Free Discontinuity Problems", Oxford University Press, 2000.

[4] B. Bourdin, G. A. FrancFort and J.-J. MARigo, "The Variational Approach to Fracture", Springer, Amsterdam, 2008.

[5] D. BUCUR and N. VARCHON, Boundary variation for a Neumann problem, Ann. Scuola Norm. Sup. Pisa Cl. Sci. (4) 29 (2000), 807-821.

[6] M. CARRIERO and A. LEACI, Existence theorem for a Dirichlet problem with free discontinuity set, Nonlinear Anal. 15 (1990), 661-677.

[7] A. Chambolle, A density result in two-dimensional linearized elasticity and applications, Arch. Ration. Mech. Anal. 167 (2003), 211-233.

[8] G. DAl MASO, G. A. FrAnCFORT and R. TOADER, Quasistatic crack growth in nonlinear elasticity, Arch. Ration. Mech. Anal. 176 (2005) 165-225.

[9] G. Dal Maso, A. Giacomini and M. Ponsiglione, A variational model for quasistatic crack growth in nonlinear elasticity: some qualitative properties of the solutions, Boll. Unione Mat. Ital. (9) 2 (2009), 371-390.

[10] G. DAl Maso and G. LAZZARONI, Quasistatic crack growth in finite elasticity with noninterpenetration, Ann. Inst. H. Poincaré Anal. Non Linéaire 27 (2010), 257-290. 
[11] G. DAL MASO and R. TOADER, A model for the quasi-static growth of brittle fracture: existence and approximation results, Arch. Ration. Mech. Anal. 162 (2002), 101-135.

[12] E. De GIORgI, M. CARRIERO and A. LEACI, Existence theorem for a minimum problem with free discontinuity set, Arch. Ration. Mech. Anal. 108 (1989), 195-218.

[13] E. DiBenedetTo, $\mathcal{C}^{1+\alpha}$ local regularity of weak solutions of degenerate elliptic equations, Nonlinear Anal. 7 (1983), 827-850.

[14] K. FAlCONER, "Fractal Geometry. Mathematical Foundations and Applications", Second edition. John Wiley \& Sons, Inc., Hoboken, NJ 2003.

[15] I. FONSECA and N. FUSCO, Regularity results for anisotropic image segmentation models, Ann. Scuola Norm. Sup. Pisa Cl. Sci. (4) 24 (1997), 463-499.

[16] G. A. FRANCFORT and G. J. LARSEN, Existence and convergence for quasi-static evolution in brittle fracture, Comm. Pure Appl. Math. 56 (2003), 1465-1500.

[17] G. A. FRANCFORT and J.-J. MARIGO, Revisiting brittle fracture as an energy minimization problem, J. Mech. Phys. Solids 46 (1998), 1319-1342.

[18] E. GiUsti, "Direct Methods in the Calculus of Variations", World Scientific Publishing, 2003.

[19] C. J. Larsen, Epsilon-stable quasi-static brittle fracture evolution, Comm. Pure Appl. Math. 63 (2010), 630-654.

Université Pierre et Marie Curie - Paris 6

CNRS, UMR 7598

Laboratoire Jacques-Louis Lions

Paris, F-75005, France

babadjian@ann.jussieu.fr

DICATAM, Sezione di Matematica

Università degli Studi di Brescia

Via Valotti, 9

25133 Brescia, Italia

alessandro.giacomini@unibs.it 